\title{
ON STABILITY OF LIQUID FLOW DOWN AN INCLINED PLANE
}

\author{
BY
}

S. M. SUN

Department of Mathematics, Virginia Polytechnic Institute and State University, Blacksburg, VA

1. Introduction. The stability of a layer of liquid flow down an inclined plane under gravity has been investigated by many authors on account of its importance in science and technology. Benjamin [1] and Yih [2] used a linear governing equation, the so-called Orr-Sommerfeld equation, to study the stability and showed that there are long surface waves that propagate downstream at about twice the mean fluid speed on the free surface. These waves are insensitive to the velocity profile and only weakly dependent on the surface tension. Their analysis is valid for small wave numbers and the waves are surface waves. However, if the steady state of the velocity is a parabola, there is another type of waves which may cause instability, in which the mean shear in the fluid is important. These are periodic waves with not very long wave-length. Lin [3] first identified such waves and made the first calculation of its neutral curves. Then De Bruin [4] calculated the neutral curves again using a corrected linearized governing equation without surface tension and Floryan et al. [5] obtained the neutral curves with surface tension. They both find that for the linearized equation, or the Orr-Sommerfeld equation, some periodic waves have lower critical Reynolds number than the long surface waves do when the inclination to the horizontal is small. The waves have wave length comparable to the mean depth of the fluid and travel more slowly than the mean speed of the fluid on the surface. The neutral curves give the stability boundary in terms of Reynolds number and wave number for the linearized equation.

In general physical situations, it is very hard to obtain any meaningful quantitative information from a direct analysis of nonlinear problems. Therefore, much of the literature on hydrodynamic stability is devoted to an analysis of the linearized problem [6, 7]. However, since the problem is in fact nonlinear, it is important to answer whether the stability or instability of the linearized problem implies the stability or instability of the full nonlinear problem. That question has not been answered mathematically for the problem considered in this paper although it is generally believed that the so-called linearization principle applies to this problem, that is, the linear stability or instability implies the nonlinear stability or instability.

Received October 31, 1994.

1991 Mathematics Subject Classification. Primary 73E05; Secondary 35Q30, 76D05.

(C)1997 Brown University 
The linearization principle was first started by Lyapunov, who studied it for nonlinear ordinary differential equations. This was later called Lyapunov's first method. Then Sattinger [8] applied this method to study hydrodynamic stability for viscous incompressible flow in a bounded region in a three-dimensional space. He showed that if such flow is disturbed slightly from any given steady state, whether the perturbations grow or decay depends upon the stability of the linearized problem with respect to the steady state, and proved rigorously that the linearization principle for the problem holds. Yudovich [9] applied semigroup theory to the problem and showed that the linearization principle holds for a wide class of partial differential equations, which includes the Navier-Stokes equations in a bounded domain as a special case. A global stability analysis for fluids in a small region was given by Serrin [10]. However, there are still very few studies that have dealt with the linearization principle for fluids with a free surface.

This paper considers the linearization principle for periodic waves, rather than the most general class of waves in the infinite domain, in a layer of fluid flow down an inclined plane with surface tension on the free surface. It has been shown formally by Benney [11] and many others that there exist long traveling waves with small amplitude under a weakly nonlinear approximation even when the linear operator has no eigenvalues with positive real part. Shen et al. [12] derived a nonlinear model equation for long waves in a layer of magnetic fluid flow down an inclined plane under the influence of a magnetic field, which also has traveling wave solutions. Therefore, one cannot expect that such solutions in an infinite domain will decay for a large time. A rigorous proof of existence of long-wave solutions of the linearized Navier-Stokes equations for fluid flow down an inclined plane was given by Shih and Shen [13] using a long-wave approximation. Beale [14] first gave an existence proof of solutions of the fully nonlinear Navier-Stokes equations with small initial data for a three-dimensional horizontal region bounded by an arbitrary rigid bottom and a free surface above. These solutions become smoother as the time tends to infinity. Then Beale and Nishida [15] showed that such solutions decay as time goes to infinity with rate $t^{-1 / 2}$ for the free surface and $t^{-1}$ for the velocity field. The slow decay rate is attributed to the existence of the eigenvalue zero of the corresponding linear operator. Joseph [16] investigated stability and instability in many situations for fluids with rigid boundaries by the energy method, and the stability of two-layer parallel shear flow with density stratification bounded by two fixed horizontal moving plates was studied rigorously by Renardy and Joseph [17]. Recently, Teramoto [18] considered a viscous fluid flow down an inclined plane in an infinite region and showed that for an arbitrary fixed $T$ and sufficiently small Reynolds number, there exists a solution of the governing equations for $0 \leq t \leq T$ if the initial condition is sufficiently close to a steady state. Then Nishida et al. [19] studied the same problem with a two-dimensional periodic motion. They obtained existence and exponential decay of solutions when the initial conditions are close to the steady state, under the assumption that the Reynolds number and the inclination angle are sufficiently small.

The present investigation explores the stability of two-dimensional periodic waves in a layer of fluid flow down an inclined plane using ideas introduced by Beale [14]. The domain of the fluid is unknown since the free surface is part of the solution. The governing equations are transformed in several steps to equations in a fixed strip domain with 
simplified linear part of the equations. Then we define appropriate Banach spaces and show that the linear operator corresponding to the linearized equations has only discrete eigenvalues. By applying a similar argument as in [14] and general elliptic operator theory, and by overcoming the difficulties arising from the unknown free surface, we show that the linear operator generates an analytic semigroup and obtain the regularity of the solutions of the linearized equations. This leads to a proof that the nonlinear terms are small compared to the linear terms on account of the form of the nonlinear terms in some carefully chosen Banach spaces: By using estimates of the solutions of the linearized equations and by the contraction mapping theorem, we show that the stability of solutions of the governing Navier-Stokes equations with small perturbations from the steady state is determined by the spectrum of the corresponding linear operator associated with the nonlinear problem. If the spectrum of the linear operator is in the complex plane with negative real part, then the perturbation decays to zero exponentially. If some of the spectrum has positive real part, however, then the perturbation grows for some initial data when time becomes large. Floryan et al. [5] have given the neutral curves of the linear operator to determine whether it has eigenvalues with positive real part. Our results show that the steady solutions of the nonlinear equations are also stable (or unstable) if the parameters are in the stable region (or unstable region) for the linear equation. For sufficiently small Reynolds number or inclination angle, it can be shown that the corresponding linear operator has no eigenvalues with nonnegative real part. Therefore, the results proved here also imply the ones in [19].

We note that the results proved here are not straightforward applications of the standard theory of evolution equations. The main difficulties are the unknown free surface and the nonstandard boundary conditions on the free surface, which involve also the time derivative of the shape of the free surface. Although the basic idea of the proof sketched in the preceding paragraph seems to be relatively simple, the actual proof is quite involved and the treatment given here is technically different from the standard stability theory. Finally, we wish to emphasize a comment by Sattinger [8]: The results may not be sufficient in actual physical cases. We have shown that if solutions of the linear problem are stable, then the steady state is stable relative to small disturbances for the nonlinear problem. In any real physical situations, the disturbances are inevitable and the question then arises whether the magnitude of the disturbances exceeds the extent of stability of the steady state. Instability might be observed in a situation where the linearized problem is stable; in such cases, a global stability analysis like the one in [10] is needed.

This paper is organized as follows. In Sec. 2, the governing Navier-Stokes equations with proper boundary conditions are given. Then these equations are transformed into a simplified form in a domain with fixed boundaries. Some Banach spaces are defined. In Sec. 3, estimates of the solutions of the time-independent linear equations are given. In Sec. 4, the existence of solutions of the modified governing equations is obtained. The stability result is given in Sec. 5 and the instability result is given in Sec. 6 .

2. Formulation. We consider two-dimensional nonlinear wave motions of an incompressible, viscous fluid of uniform density on a straight, inclined plane. A rectangular 
coordinate system $\left(x_{1}^{*}, x_{2}^{*}\right)=\left(x^{*}, z^{*}\right)$ is chosen so that $z^{*}=x_{2}^{*}=0$ coincides with the steady free surface of the liquid and $z^{*}=-h^{*}$ is the equation of the rigid bottom. If a surface wave is generated by some initial disturbance, then the motion of the flow is governed by the two-dimensional Navier-Stokes equations,

$$
\begin{aligned}
\nabla^{*} \cdot \bar{q}^{*} & =0 \\
\rho^{*} \frac{D \bar{q}^{*}}{D t^{*}} & =-\nabla^{*} p^{*}+\rho^{*} \bar{g}^{*}+\mu\left(\nabla^{*}\right)^{2} \bar{q}^{*},
\end{aligned}
$$

subject to the boundary conditions at $\xi^{*}=-z^{*}+\zeta^{*}\left(x^{*}, t^{*}\right)=0$,

$$
\begin{aligned}
\frac{D \xi^{*}}{D t^{*}} & =0 \\
\sum_{k=1}^{2} \sigma_{i k}^{*} n_{k}^{*} & =T\left(R_{1}^{-1}+R_{2}^{-1}\right) n_{i}, \quad i=1,2
\end{aligned}
$$

and that $z^{*}=-h^{*}$,

$$
\bar{q}^{*}=(0,0)
$$

where $\nabla^{*}=\left(\partial / \partial x^{*}, \partial / \partial z^{*}\right), D / D t^{*}=\partial / \partial t^{*}+\bar{q}^{*} \cdot \nabla^{*}, \bar{q}^{*}=\left(q_{1}^{*}, q_{2}^{*}\right)=\left(u^{*}, v^{*}\right)$ is the velocity vector, $\rho^{*}$ the density of the fluid, $p^{*}$ the pressure, $\bar{g}^{*}=(g \sin \theta,-g \cos \theta)$ the constant gravity with gravitational acceleration constant $g>0$ and the inclination angle $0<\theta<\pi / 2, \mu$ the constant viscosity coefficient, $\sigma_{i k}^{*}=\mu\left(\partial q_{i}^{*} / \partial x_{k}^{*}+\partial q_{k}^{*} / \partial x_{i}^{*}\right)-p^{*} \delta_{i k}$ the stress tensor, $\left(n_{1}^{*}, n_{2}^{*}\right)=\left(\partial \zeta^{*} / \partial x^{*},-1\right)$ the normal vector at the free surface $\xi^{*}=0$, $T$ the constant coefficient of surface tension, and

$$
R_{1}^{-1}+R_{2}^{-1}=\zeta_{x^{*} x^{*}}^{*}\left(1+\left(\zeta_{x^{*}}^{*}\right)^{2}\right)^{-3 / 2}
$$

Now we nondimensionalize the equations by measuring $\zeta^{*}, x^{*}, z^{*}$ in units of $h^{*}, \bar{q}^{*}$ in units of $\left(g h^{*}\right)^{1 / 2}, p^{*}$ in units of $\rho^{*} g h^{*}, t^{*}$ in units of $\left(h^{*} / g\right)^{1 / 2}$, and define

$$
R=\mu^{-1} \rho^{*} g^{1 / 2}\left(h^{*}\right)^{3 / 2}, \quad \tau=T \mu^{-1} g^{-1 / 2}\left(h^{*}\right)^{-1 / 2},
$$

where $R$ is referred to as the Reynolds number of the fluid. Thus the equations (1) to (5) are transformed to

$$
\begin{gathered}
\widehat{\nabla} \cdot \widehat{U}=0 \\
\widehat{U}_{t}+\widehat{U} \cdot \widehat{\nabla} \widehat{U}=-\widehat{\nabla} \hat{p}+R^{-1}\left(\widehat{\nabla}^{2} \widehat{U}\right)+\widehat{H}
\end{gathered}
$$

with boundary conditions at $\hat{z}=\eta(\hat{x}, t)$,

$$
\begin{aligned}
\left(R \hat{p}-2 \hat{u}_{1 \hat{x}}\right) \eta_{\hat{x}}+\hat{u}_{1 \hat{z}}+\hat{u}_{2 \hat{x}} & =-\tau\left(\widehat{R}_{1}^{-1}+\widehat{R}_{2}^{-1}\right) \eta_{\hat{x}}, \\
R \hat{p}-2 \hat{u}_{2 \hat{z}}+\left(\hat{u}_{1 \hat{z}}+\hat{u}_{2 \hat{x}}\right) \eta_{\hat{x}} & =-\tau\left(\widehat{R}_{1}^{-1}+\widehat{R}_{2}^{-1}\right), \\
\eta_{t}+\hat{u}_{1} \eta_{\hat{x}}-\hat{u}_{2} & =0
\end{aligned}
$$


at $z=-1$,

$$
\hat{u}_{1}=\hat{u}_{2}=0,
$$

where $\hat{\nabla}=(\partial / \partial \hat{x}, \partial / \partial \hat{z}), \widehat{U}=\left(\hat{u}_{1}, \hat{u}_{2}\right),(\hat{x}, \hat{z}), \eta$, and $\hat{p}$ are nondimensional variables for gradient, velocity, independent variables, displacement of the free surface from $\hat{z}=0$, and pressure, respectively, and $\widehat{H}=(\sin \theta,-\cos \theta)$,

$$
\widehat{R}_{1}^{-1}+\widehat{R}_{2}^{-1}=\eta_{\hat{x} \hat{x}}\left(1+\left(\eta_{\hat{x}}\right)^{2}\right)^{-3 / 2} .
$$

Equations (8) to (13) have a steady state

$$
U_{0}=\left((R \sin \theta / 2)\left(1-\hat{z}^{2}\right), 0\right), \quad \hat{p}_{0}=-\hat{z} \cos \theta, \quad \eta_{0}=0 .
$$

In the following, we shall consider the existence and stability of solutions of (8) to (13), where the initial values of $\widehat{U}, \hat{p}$, and $\eta$ are close to (14). Let

$$
\widetilde{U}=\left(\tilde{u}_{1}, \tilde{u}_{2}\right)=\widehat{U}-U_{0}, \quad p=\hat{p}-\hat{p}_{0},
$$

and $\eta(\hat{x}, t)$ be the perturbation from the steady state (14). Equations (8) to (13) are then transformed to

$$
\begin{gathered}
\hat{\nabla} \cdot \widetilde{U}=0, \\
\widetilde{U}_{t}+U_{0} \cdot \widehat{\nabla} \widetilde{U}+\widetilde{U} \cdot \hat{\nabla} U_{0}+\widetilde{U} \cdot \widehat{\nabla} \widetilde{U}=-\widehat{\nabla} p+R^{-1}(\widehat{\nabla})^{2} \widetilde{U}
\end{gathered}
$$

with boundary conditions at $\hat{z}=\eta(\hat{x}, t)$,

$$
\begin{aligned}
& \left(R p-R \eta \cos \theta-2 \widehat{u}_{1 \hat{x}}\right) \eta_{\hat{x}}+\tilde{u}_{1 \hat{z}}+\tilde{u}_{2 \hat{x}}-R \eta \sin \theta=-\tau\left(\widehat{R}_{1}^{-1}+\widehat{R}_{2}^{-1}\right) \eta_{\hat{x}} \\
& R p-R \eta \cos \theta-2 \tilde{u}_{2 \hat{z}}+\left(\tilde{u}_{1 \hat{z}}-R \eta \sin \theta+\tilde{u}_{2 \hat{x}}\right) \eta_{\hat{x}}=-\tau\left(\widehat{R}_{1}^{-1}+\widehat{R}_{2}^{-1}\right), \\
& \eta_{t}+(R \sin \theta / 2) \eta_{\hat{x}}+\left(\tilde{u}_{1}-(R \sin \theta / 2) \eta^{2}\right) \eta_{\hat{x}}-\tilde{u}_{2}=0
\end{aligned}
$$

at $\hat{z}=-1$,

$$
\widetilde{U}=0
$$

Then we let

$$
(\tilde{x}, \tilde{z})=(\hat{x}+(R \sin \theta / 2) t, \hat{z})
$$

to remover $(R \sin \theta / 2) \eta_{\hat{x}}$ in (20) for the sake of convenience, which will be seen later. Equations (17) and 20 are changed to

$$
\begin{aligned}
\widetilde{U}_{t}+\widetilde{U}_{0} \cdot \widetilde{\nabla} \tilde{U}+\widetilde{U} \cdot \widetilde{\nabla} U_{0}+\widetilde{U} \cdot \widetilde{\nabla} \widetilde{U} & =-\widetilde{\nabla}_{p}+R^{-1}(\widetilde{\nabla})^{2} \widetilde{U} \\
\eta_{t}+\left(\tilde{u}_{1}-(R \sin \theta / 2) \eta^{2}\right) \eta_{\hat{x}}-\tilde{u}_{2} & =0,
\end{aligned}
$$

and other equations are the same except for changing $(\hat{x}, \hat{z})$ to $(\tilde{x}, \tilde{z})$, where $\widetilde{U}_{0}=$ $\left(-(R \sin \theta / 2) \hat{z}^{2}, 0\right)$. Since we are only interested in the periodic solutions of (16) to (21) in $\hat{x}$, the change of variables (22) will not affect the function spaces defined later. 
Since $\tilde{z}=\eta(\tilde{x}, t)$ for the free surface is part of the solutions of (16) to (21) and the domain of the fluid is unknown, in the following we use a transform introduced in [1] to reduce the unknown domain to a fixed strip domain. Given $\eta(\tilde{x}, t)$, let $\bar{\eta}(\tilde{x}, z, t)$ be an extension to $-1 \leq z \leq 0$ such that $\bar{\eta}(\tilde{x}, 0, t)=\eta(\tilde{x}, t)$. Such an extension in certain Banach spaces will be introduced at the end of this section. Now let $\theta(x, z, t)$ be a transformation that maps the domain bounded by $-1 \leq z \leq 0$ to a domain bounded by $-1 \leq \tilde{z} \leq \eta(\tilde{x}, t)$, such that

$$
\theta(x, z, t)=(\tilde{x}, \tilde{z})=\left(\tilde{x}_{1}, \tilde{x}_{2}\right)=(x, \bar{\eta}(x, z, t)+z(1+\bar{\eta}(x, z, t)))
$$

and denote the Jacobi matrix

$$
\widetilde{B}=\frac{\partial\left(x_{1}, x_{2}\right)}{\partial\left(\tilde{x}_{1}, \tilde{x}_{2}\right)}=\frac{\partial(x, z)}{\partial(\tilde{x}, \tilde{z})}=\left(\tilde{b}_{i j}\right)_{2 \times 2} .
$$

Since (16) does not hold under such a transformation, we change $\widetilde{U}$ to $\widetilde{V}=\left(\tilde{v}_{1}, \tilde{v}_{2}\right)$ by

$$
\begin{aligned}
\widetilde{U}=\left(\tilde{u}_{1}, \tilde{u}_{2}\right) & =\left(1+\bar{\eta}+\bar{\eta}_{z}(1+z)\right)^{-1}\left(\tilde{v}_{1}, \tilde{v}_{1} \bar{\eta}_{x}(1+z)+\left(1+\bar{\eta}+\bar{\eta}_{z}(1+z)\right) \tilde{v}_{2}\right) \\
& =\widetilde{A} \widetilde{V}=\left(\tilde{a}_{i j} \tilde{v}_{j}\right)
\end{aligned}
$$

where

$$
\widetilde{A}=\left(1+\bar{\eta}+\bar{\eta}_{z}(1+z)\right)^{-1}\left(\begin{array}{cc}
1 & 0 \\
(1+z) \bar{\eta}_{x} & 1+\bar{\eta}+(1+z) \bar{\eta}_{z}
\end{array}\right)=\left(\tilde{a}_{i j}\right)_{2 \times 2} .
$$

From (16), (23), (18), (19), (24), and (21), we have

$$
\begin{gathered}
\nabla \cdot \widetilde{V}=0, \\
\widetilde{A} \widetilde{V}_{t}+\widetilde{A}_{t} \widetilde{V}+\widetilde{A}\left(\tilde{b}_{2 j} \partial_{j} \widetilde{V}\right)(1+z) \bar{\eta}_{t}+\widetilde{U}_{0}^{(1)} \cdot(\widetilde{B} \nabla)(\widetilde{A} \widetilde{V}) \\
+(\widetilde{A} \widetilde{V}) \cdot\left((\widetilde{B} \nabla) U_{0}^{(1)}\right)+(\widetilde{A} \widetilde{V}) \cdot(\widetilde{B} \nabla)(\widetilde{A} \widetilde{V}) \\
=-(\widetilde{B} \nabla) p+R^{-1}(\widetilde{B} \nabla)^{2}(\widetilde{A} \widetilde{V}),
\end{gathered}
$$

with boundary conditions at $z=0$,

$$
\begin{gathered}
\eta_{t}-\tilde{v}_{2}-(R \sin \theta / 2) \eta^{2} \eta_{x}=0 \\
\left(R(p-\eta \cos \theta)-2 \tilde{b}_{1 i} \partial_{i}\left(\tilde{a}_{1 j} \tilde{v}_{j}\right)\right) \eta_{x}+\tilde{b}_{2 i}\left(\tilde{a}_{1 j} \tilde{v}_{j}\right) \\
+\tilde{b}_{1 i} \partial_{i}\left(\tilde{a}_{2 j} \tilde{v}_{j}\right)-R \eta \sin \theta=-\tau\left(R_{1}^{-1}+R_{2}^{-1}\right) \eta_{x}, \\
R(p-\eta \cos \theta)-2 \tilde{b}_{2 i} \partial_{i}\left(\tilde{a}_{2 j} \tilde{v}_{j}\right)+\left(\tilde{b}_{2 i} \partial_{i}\left(\tilde{a}_{1 j} \tilde{v}_{j}\right)\right. \\
\left.\tilde{b}_{1 i} \partial_{i}\left(\tilde{a}_{2 j} \tilde{v}_{j}\right)-R \eta \sin \theta\right) \eta_{x}=-\tau\left(R_{1}^{-1}+R_{2}^{-1}\right),
\end{gathered}
$$

at $z=-1$,

$$
\widetilde{V}=0,
$$


where $\nabla=(\partial / \partial x, \partial / \partial z)$ and $U_{0}^{(1)}, \widetilde{U}_{0}^{(1)}$ are the corresponding functions of $U_{0}$ and $\widetilde{U}_{0}$ under the transformation and

$$
R_{1}^{-1}+R_{2}^{-1}=\eta_{x x}\left(1+\eta_{x}^{2}\right)^{-3 / 2}
$$

We note that $(28)$ to $(33)$ are equivalent to $(16),(23),(18),(19),(24)$, and (21) if $\widetilde{A}, \widetilde{B}$ are invertible in some spaces and the transformation (22) makes (30) a simple form. We multiply both sides of (29) by the inverse of $\widetilde{A}$ and move all the linear terms to the left-hand sides of (28) to (33) and the nonlinear terms to the right except (30). In order to remove the nonlinear term in (30), we use the transformation

$$
\widetilde{V}=U-U_{1}
$$

where

$$
U_{1}=\left(\begin{array}{c}
(R \sin \theta / 2)\left(1+\bar{\eta}+\bar{\eta}_{z}(1+z)\right)\left(z^{2}-(\bar{\eta}+z(1+\bar{\eta}))^{2}\right)+R \sin \theta(z+1) z \bar{\eta} \\
(R \sin \theta / 2) \bar{\eta}_{x}(1+z)\left((\bar{\eta}+z(1+\bar{\eta}))^{2}-z^{2}\right)
\end{array}\right) .
$$

It is easy to check that $\nabla \cdot U_{1}=0$ and $U_{1}=0$ at $z=-1$. Since $U_{1}$ has only nonlinear terms in $\eta$, it has no contributions to the linear terms in (28) to (33). Finally (28) to (33) are transformed to

$$
\begin{gathered}
\nabla \cdot U=0 \\
U_{t}+\widetilde{U}_{0} \cdot \nabla U+U \cdot \nabla U_{0}+\nabla p-R^{-1} \nabla^{2} U=F(U, \eta, p),
\end{gathered}
$$

at $z=0$,

$$
\begin{aligned}
R^{-1}\left(u_{1 z}+u_{2 x}\right)-\eta \sin \theta & =f_{1}(U, \eta, p), \\
p-2 R^{-1} u_{2 z}+\beta \eta_{x x}-\eta \cos \theta & =f_{2}(U, \eta, p), \\
\eta_{t}-u_{2} & =0
\end{aligned}
$$

at $z=-1$,

$$
U=0 \text {, }
$$

where $\widetilde{U}_{0}=\left(-(R \sin \theta / 2) z^{2}, 0\right), U_{0}=\left((R \sin \theta / 2)\left(1-z^{2}\right), 0\right), \beta=\tau / R$, and $F, f_{1}, f_{2}$ are nonlinear functions of $U, \eta, p$ and their derivatives. If the temporal derivative of a function is considered as a second-order derivative of spatial variables, then the highest derivative in $F$ is third for $\bar{\eta}$, second for $U$, and first for $p$, while the highest derivative in $f_{1}, f_{2}$ is second for $\eta$, first for $U$, and zeroth for $p$.

Before we show the proof of the existence and stability of solutions of (34) to (39), the following Banach spaces have to be defined. Let

$$
\Omega=[0,2 \pi \kappa] \times[-1,0]=\Gamma \times[-1,0]=\{(x, z) \mid 0 \leq x \leq 2 \pi \kappa,-1 \leq z \leq 0\}
$$

$H_{p}^{r}(\Omega)\left(H_{p}^{r}(\Gamma)\right)$ be the usual Sobolev space of periodic functions in $x$ with derivatives in $L^{2}(\Omega)\left(L^{2}(\Gamma)\right)$ to the order $r$ if $r$ is a nonnegative integer, or the usual generalization 
otherwise, where $\kappa$ is a fixed positive constant. In the following, the functions are always periodic with period $2 \pi \kappa$. If $r, s$ are nonnegative real numbers, define

$$
H_{p}^{r, s}(\Omega \times[0, T])=H^{0}\left((0, T) ; H_{p}^{r}(\Omega)\right) \cap H^{s}\left((0, T) ; H_{p}^{0}(\Omega)\right),
$$

with a similar definition for $H_{p}^{r, s}(\Gamma \times[0, T])$. For $f \in H_{p}^{r, s}$, the norm is defined as

$$
\|f\|_{H_{p}^{r, s}}^{2}=\int_{0}^{T}\|f(\cdot, s)\|_{H_{p}^{r}}^{2} d s+\int\|f(x, \cdot)\|_{H^{s}(0, T)}^{2} d x,
$$

where it should be clear from the context whether scalar or vector-valued functions are meant. Let $K^{r}(\Omega \times[0, T])=H_{p}^{r, r / 2}(\Omega \times[0, T])$ and $K_{0}^{r}(\Omega \times[0, T])=\left\{u \in K^{r}(\Omega \times\right.$ $[0, T] \mid \partial_{t}^{k} u(\cdot, 0)=0$ for $\left.2 k<r-1\right\}$ with corresponding definitions for $K^{r}(\Gamma \times[0, T])$ and $K_{0}^{r}(\Gamma \times[0, T])$. Also denote $\mathbf{R}=(-\infty,+\infty)$ and $\mathbf{R}^{+}=[0,+\infty)$. Now define $\bar{\eta}(x, z, t)$ from $\eta(x, t)$ as follows. If $\eta(x, t) \in H_{p}^{r}(\Gamma)$ for each $t \geq 0$, then $\eta(x, t)=$ $\sum_{n=-\infty}^{+\infty} c_{n}(t) \exp (i n x / \kappa)$ where $\sum_{n=-\infty}^{+\infty}\left|c_{n}(t)\right|^{2}\left(|n|^{2 r}+1\right)<+\infty$. Let

$$
\bar{\eta}(x, z, t)=\sum_{n=-\infty}^{+\infty} c_{n}(t) \exp (|n| z+i n x / \kappa), \quad \text { for }-1 \leq z \leq 0,
$$

which implies $\bar{\eta}(x, 0, t)=\eta(x, t)$ and $\bar{\eta} \in H_{p}^{r+1 / 2}(\Omega)$ for each $t$.

3. Estimates for solutions of linear equations. In this section, we shall obtain estimates of solutions of the following time-independent linear equations: in $\Omega=I \times$ $(-1,0)$,

$$
\begin{gathered}
\nabla \cdot U=0 \\
\lambda U+\widetilde{U}_{0} \cdot \nabla U+U \cdot \nabla U_{0}+\nabla p-R^{-1} \nabla^{2} U=G(x, z) ;
\end{gathered}
$$

at $\Gamma=I \times\{z=0\}$,

$$
\begin{aligned}
\lambda \eta-u_{2} & =g_{0}(x), \\
R^{-1}\left(u_{1 z}+u_{2 x}\right)-\eta \sin \theta & =g_{1}(x), \\
p-2 R^{-1} u_{2 z}+\beta \eta_{x x}-\eta \cos \theta & =g_{2}(x) ;
\end{aligned}
$$

at $B=I \times\{z=-1\}$

$$
u_{1}=u_{2}=0,
$$

where $\lambda$ is a complex parameter. By $(40)$, it is natural to project the function $U$ onto a subspace of $L^{2}(\eta)$ whose functions satisfy (40) and (45). From the identity

$$
\int_{\Omega}[U \cdot \nabla \phi+(\nabla \cdot U) \phi] d \Omega=\int_{\partial \Omega}(U \cdot \eta) \phi d s,
$$

where $n$ is the normal vector of $\partial \Omega$, we have that a vector field $U \in H_{p}^{1}(\Omega)$ satisfies (40) and $U \cdot n=0$ on $B$ if and only if $U$ is $L^{2}$-orthogonal to $\nabla \phi$ for any $\phi \in H_{p}^{1}(\Omega)$ and $\phi=0$ on $\Gamma$. Therefore, we define the projection $P$ on $L^{2}(\Omega)$ orthogonal to

$$
\left\{\nabla \phi: \phi \in H_{p}^{1}(\Omega), \quad \phi=0 \text { on } \Gamma\right\} .
$$

For the sake of convenience, we first let $g_{1}(x)=g_{2}(x)=0$ in (43) and (44). By the definition of $P$, we have 
LEMma 1. $P$ is a bounded operator on $H_{p}^{r}(\Omega)$ and on $K^{r}\left(\Omega \times \mathbf{R}^{+}\right)$for $r \geq 0$. If $\phi \in H_{p}^{1}(\Omega), P(\nabla \phi)=\nabla \pi_{1}$ where

$$
\nabla^{2} \pi_{1}=0 \text { in } \Omega, \quad \pi_{1}=\phi \text { on } \Gamma, \quad \text { and } \pi_{z}=0 \text { on } B .
$$

The proof of the lemma can be found in [1]. We apply $P$ to Eq. (41) to have

$$
\lambda U+P\left(\widetilde{U}_{0} \cdot \nabla U+U \cdot \nabla U_{0}\right)+\nabla p_{1}+\nabla p_{2}-R^{-1} P\left(\nabla^{2} U\right)=P G
$$

where $\nabla^{2} p_{i}=0, p_{i z}=0$ on $B, i=1,2$, and $p_{1}=2 R^{-1} u_{2 z}, p_{2}=\eta \cos \theta-\beta \eta_{x x}$ on $\Gamma$. Let $E$ be an operator from a scalar-valued function on $\Gamma$ to $P L^{2}\left(\Omega, \mathbf{R}^{2}\right)$ such that if $h$ is a function on $\Gamma, E h=\nabla q$ where $q$ satisfies

$$
\nabla^{2} q=0 \text { in } \Omega, \quad g=h \text { on } \Gamma, \quad \text { and } q_{z}=0 \text { on } B .
$$

We note that $\left.u_{2}\right|_{\Gamma}$ is well defined if $U \in H^{r}(\Omega)$ for $r>1 / 2$. Now let us define linear operators $A, B$ as follows:

$$
\begin{aligned}
& B U=P\left(\widetilde{U}_{0} \cdot \nabla U+U \cdot \nabla U_{0}\right) \\
& A U=R^{-1} P\left(\nabla^{2} U\right)-E\left(2 R^{-1} u_{2 z}\right) .
\end{aligned}
$$

Then (47) becomes

$$
\lambda U+B U-A U+E\left(\eta \cos \theta-\beta \eta_{x x}\right)=P G .
$$

By (42), we have

$$
\lambda \eta-\left.u_{2}\right|_{\Gamma}=g_{0}(x) .
$$

In the following we study the operator defined by

$$
\mathcal{A}\left(\begin{array}{l}
U \\
\eta
\end{array}\right)=\left(\begin{array}{c}
A U-B U-E\left(\eta \cos \theta-\beta \eta_{x x}\right) \\
\left.u_{2}\right|_{\Gamma}
\end{array}\right),
$$

with domain

$$
\begin{array}{r}
\mathcal{D}(\mathcal{A})=\left\{\bar{U}=\left(\begin{array}{l}
U \\
\eta
\end{array}\right) \mid U \in P L^{2}(\Omega) \cap H_{p}^{2}(\Omega), \eta \in H_{p}^{5 / 2}(\Gamma),\right. \\
\left.R^{-1}\left(u_{1 z}+u_{2 x}\right)-\eta \sin \theta=0 \text { on } \Gamma, U=0 \text { on } B\right\} .
\end{array}
$$

Obviously $\mathcal{D}(\mathcal{A})$ is dense and closed in $L_{p}^{2}(\Omega) \times L_{p}^{2}(\Gamma)$. Define a Hilbert space

$$
H=\left\{\bar{U}=\left(\begin{array}{l}
U \\
\eta
\end{array}\right) \mid U \in P L^{2}(\Omega), \eta \in H_{p}^{1}(\Gamma)\right\},
$$

with inner product

$$
(\bar{U}, \bar{V})_{H}=\int_{\Omega} U \cdot V^{*} d \Omega+\int_{\Gamma}\left(\eta \zeta^{*} \cos \theta+\beta \eta_{x} \zeta_{x}^{*}\right) d \Gamma
$$


where $f^{*}$ is the complex conjugate of $f$. Thus (40) to (45) are transformed to

$$
(\lambda I-\mathcal{A}) \bar{U}=\left(\begin{array}{c}
P G \\
g_{0}
\end{array}\right)=\bar{G}
$$

By assuming $\bar{G} \in H$, we try to find a solution $\bar{U}$ of (52) so that $\bar{U} \in \mathcal{D}(\mathcal{A})$ for some $\lambda \in \mathbf{C}$. Thus we have to solve the following equations:

$$
\begin{gathered}
\lambda U-\left(A U-B U-E\left(\eta \cos \theta-\beta \eta_{x x}\right)\right)=P G, \\
\lambda \eta-\left.u_{2}\right|_{\Gamma}=g_{0},
\end{gathered}
$$

with $\bar{G}=\left(P G, g_{0}\right)^{\mathrm{T}} \in H$ where $\mathrm{T}$ denotes the transpose of a matrix. To have a solution of (52), we introduce an identity. If $U, V \in H_{p}^{2}(\Omega), p \in H_{p}^{1}(\Omega)$, and $\nabla \cdot U=0$, then

$$
\int_{\Omega}\left(-R^{-1} \nabla^{2} U+\nabla p\right) \cdot V^{*} d \Omega=\langle U, V\rangle+\int_{\partial \Omega} S(U, p) V^{*} d s-\int_{\Omega} p\left(\nabla \cdot V^{*}\right) d \Omega
$$

where

$$
\langle U, V\rangle=\left(2 R^{-1}\right) \int_{\Omega} \sum_{i, j=1}^{2}\left(u_{i, j}+u_{j, i}\right)\left(v_{i, j}^{*}+v_{j, i}^{*}\right) d \Omega
$$

and

$$
S(U, p)_{i}=p n_{i}-R^{-1} \sum_{j=1}^{2}\left(u_{i, j}+u_{j, i}\right) n_{j} \quad \text { for } i=1,2
$$

with $n$ the normal vector on $\partial \Omega$. Therefore from (55) if $\nabla \cdot V=0$ in $\Omega$ and $\left.V\right|_{B}=0$, (51) can be written as

$$
\begin{aligned}
(\lambda U- & \left.\left(A U-B U-E\left(\eta \cos \theta-\beta \eta_{x x}\right)\right), V\right)_{L^{2}(\Omega)} \\
= & \int_{\Omega}(\lambda U+B U) \cdot V^{*} d \Omega+\langle U, V\rangle \\
& \quad+\int_{\Omega}\left(-\eta v_{1}^{*} \sin \theta+\eta v_{2}^{*}+\beta \eta_{x} v_{2 x}^{*}\right) d \Gamma \\
= & (P G, V)_{L^{2}(\Omega)} .
\end{aligned}
$$

By $\lambda \eta-u_{2}=g_{0}$ on $\Gamma$, we have $\eta=\left(u_{2}+g_{0}\right) / \lambda$ if $\lambda \neq 0$. Therefore, the solution of (53) and (54) satisfies

$$
\begin{array}{rl}
\int_{\Omega}(\lambda U+B U) \cdot V^{*} & d \Omega+\langle U, V\rangle+\lambda^{-1} \int_{\Gamma}\left(-u_{2} v_{1}^{*} \sin \theta+u_{2} v_{2}^{*} \cos \theta+\beta u_{2 x} v_{2 x}^{*}\right) d \Gamma \\
& =\int_{\Omega} P G \cdot V^{*} d \Omega+\lambda^{-1} \int_{\Omega}\left(g_{0} v_{1}^{*} \sin \theta-g_{0} v_{2}^{*} \cos \theta-\beta g_{0 x} v_{2 x}^{*}\right) d \Gamma
\end{array}
$$

for all $V \in H_{p}^{1}(\Omega)$ with $\nabla \cdot V=0$ and $\left.V\right|_{B}=0$. We call the solution of (57) a generalized solution of (53) and (54). In order to find a solution of (57), we denote $\mathcal{L}_{\lambda}(U, V)$ as the 
form of the left-hand side of (57) and $\mathcal{H}(\bar{G}, V)$ as the right-hand side of (57). Then (57) becomes $\mathcal{L}_{\lambda}(U, V)=\mathcal{H}(\bar{G}, V)$. If we use the inner product

$$
(U, V)=\int_{\Omega} \sum_{\substack{j=0,1 \\ i=1,2}}\left(\frac{\partial^{j} U}{\partial x_{i}^{j}} \cdot \frac{\partial^{j} V^{*}}{\partial x_{i}^{j}}\right) d \Omega+\int_{\Gamma}\left(u_{2} v_{2}^{*}+u_{2 x} v_{2 x}^{*}\right) d \Gamma
$$

to define a Hilbert space $\mathcal{S}$, then $\mathcal{L}_{\lambda}(U, V)$ is a bounded bilinear form in $\mathcal{S}$. Since $\bar{G} \in H$, $\mathcal{H}(\bar{G}, V)$ is a bounded functional in $\mathcal{S}$. If $V=U$ in (57),

$$
\begin{aligned}
\mathcal{L}_{\lambda}(U, U)=\int_{\Omega}\left(\lambda|U|^{2}+(B U) \cdot U^{*}\right) d \Omega+\langle U, V\rangle \\
\quad+\lambda^{-1} \int_{\Gamma}\left(-u_{2} u_{1}^{*} \sin \theta+\left|u_{2}\right|^{2} \cos \theta+\beta\left|u_{2 x}\right|^{2}\right) d \Gamma .
\end{aligned}
$$

However, because $\langle U, U\rangle+\|U\|_{L^{2}(\Omega)}^{2}$ is equivalent to the $H^{1}$-norm in $\Omega$,

$$
\left|\int_{\Omega}(B U) \cdot U^{*} d \Omega\right| \leq(1 / 4)\langle U, U\rangle+C_{0}\|U\|_{L^{2}(\Omega)}^{2}
$$

where $C_{0}$ is a fixed constant. Let $\lambda$ be a positive real number such that

$$
\left|\lambda^{-1} \int_{\Gamma}\left(-u_{2} u_{1}^{*} \sin \theta\right) d \Gamma\right| \leq(1 / 4)\langle U, U\rangle+C_{1}\|U\|_{L^{2}(\Omega)} .
$$

Thus there exists a $\lambda_{0}>0$ such that for all $\operatorname{Re} \lambda \geq \lambda_{0}$

$$
\operatorname{Re}\left(\mathcal{L}_{\lambda}(U, U)\right) \geq C\left(\int_{\Omega} \sum_{\substack{j=0,1 \\ i=1,2}}\left|\frac{\partial^{j} U}{\partial x_{i}^{j}}\right|^{2} d \Omega+\int_{\Gamma}\left(\left|u_{2}\right|^{2}+\left|u_{2 x}\right|^{2}\right) d \Gamma\right)
$$

where $C$ is a small positive number. Therefore $\mathcal{L}_{\lambda}(U, U)$ is coercive in $\mathcal{S}$. By the LaxMilgram Theorem, there exists a unique solution $U$ satisfying (57) and $U \in H_{p}^{1}(\Omega)$ and $\left.u_{2}\right|_{\Gamma} \in H_{p}^{1}(\Gamma)$ with its norm bounded by $\|P G\|_{L_{p}^{2}(\Omega)}+\left\|g_{0}\right\|_{H_{p}^{1}(\Gamma)}$, which implies that $\eta=\left(u_{2}+g_{0}\right) / \lambda \in H_{p}^{1}(\Gamma)$. Therefore, given $\bar{G}$ in (52) with $\bar{G} \in H$, there exists a generalized solution for (52) and for $\operatorname{Re} \lambda \geq \lambda_{0},(\lambda I-\mathcal{A})$ is invertible in $H$, i.e., $(\lambda I-\mathcal{A})^{-1}$ exists and $(\lambda I-\mathcal{A})^{-1} \bar{G}=\bar{U}$, and $\bar{U}$ is bounded in $H$ by $\bar{G}$. Here $U$ also has first-order derivatives in $L_{p}^{2}(\Omega)$.

Now we study the spectrum of $\mathcal{A}$. Let $\mathcal{B}=\left(\lambda_{0} I-\mathcal{A}\right)^{-1}$ be a bounded operator from $H$ to $H$. We shall prove the following lemma.

Lemma 2. The operator $\mathcal{B}$ is a compact operator from $H$ to $H$.

Proof. First we need the following identity. If $\bar{U}=(U, \eta)^{\mathrm{T}}=\mathcal{B} \bar{G}$, then

$$
\begin{aligned}
\int_{\Omega}\left(\lambda_{0} U+B U\right) \cdot V^{*} d \Omega+\langle U, V\rangle & +\lambda_{0}^{-1} \int_{\Gamma}\left(-u_{2} v_{1}^{*} \sin \theta+u_{2} v_{2}^{*} \cos \theta+\beta\left(u_{2 x}+g_{2 x}\right) v_{2 x}^{*}\right) d \Gamma \\
& =\int_{\Omega} P G \cdot V^{*} d \Omega-\lambda_{0}^{-1} \int_{\Gamma}\left(-g_{0} v_{1}^{*} \sin \theta+g_{0} v_{2}^{*} \cos \theta\right) d \Gamma
\end{aligned}
$$


where $\bar{G}=\left(P G, g_{0}\right)^{\mathrm{T}} \in H$ and $V \in H_{p}^{1}(\Omega)$ with $\nabla \cdot V=0,\left.V\right|_{B}=0$. Let $\bar{G}_{n}=\left(G_{n}, g_{n}\right)^{\mathrm{T}}$, $n=1,2,3, \ldots$, be a bounded sequence in $H$ and $\bar{U}_{n}=\left(U_{n}, \eta_{n}\right)^{\mathrm{T}}=\mathcal{B} \bar{G}_{n}$. Let $V=U_{n}$ in (58). By the choice of $\lambda_{0}, U_{n}$ and the first-order derivatives of $U_{n}$ are bounded in $L_{p}^{2}(\Omega)$ and $u_{n, 2},\left(u_{n, 2}\right)_{x}$ are bounded in $L_{p}^{2}(\Gamma)$. From the definition of $\mathcal{B}$, we have $\eta_{n}=\left(g_{n}+u_{2, n}\right) / \lambda_{0}$. Thus $\eta_{n}$ and $\left(\eta_{n}\right)_{x}$ are bounded in $L^{2}(\Gamma)$. Therefore, there exists a subsequence $\bar{U}_{n_{k}}$ such that $U_{n_{k}}$ is convergent in $L_{p}^{2}(\Omega)$ and $\eta_{n_{k}}$ is convergent in $H_{p}^{1 / 2}(\Gamma)$. Next we need to prove $\eta_{n_{k}}$ is convergent in $H_{p}^{1}(\Gamma)$. We rewrite (58) as

$$
\begin{aligned}
\beta \int_{\Omega} \eta_{x} v_{2 x}^{*} d \Gamma & =-\langle U, V\rangle-\int_{\Omega}\left(\lambda_{0} U+B U-P G\right) \cdot V^{*} d \Omega \\
& -\lambda_{0}^{-1} \int_{\Gamma}\left(-u_{2} v_{1}^{*} \sin \theta+u_{2} v_{2}^{*} \cos \theta-g_{0} v_{1}^{*} \sin \theta+g_{0} v_{2}^{*} \cos \theta\right) d \Gamma .
\end{aligned}
$$

By applying (59) for $\bar{U}_{n_{k}}-\bar{U}_{m_{k}}=\mathcal{B}\left(\bar{G}_{n_{k}}-\bar{G}_{m_{k}}\right)$, we have

$$
\beta \int_{\Omega}\left(\eta_{n_{k}}-\eta_{m_{k}}\right)_{x} v_{2 x}^{*} d \Gamma=J\left(\bar{U}_{n_{k}}, \bar{U}_{m_{k}}, V, \bar{G}_{n_{k}}, \bar{G}_{m_{k}}\right) .
$$

We choose $V=\left(v_{1}, v_{2}\right)$ as follows. For a function $h(x) \in H_{p}^{1 / 2}(\Gamma)$ and

$$
h(x)=\sum_{n=-\infty}^{+\infty} c_{n} \exp (i n x / \kappa)
$$

let

$$
\begin{aligned}
& v_{1}(x, z)=-\sum_{\substack{n=-\infty \\
n \neq 0}}^{+\infty} c_{n}\left(\varphi^{\prime}(z)+\varphi(z)|n|\right)(\kappa / i n) \exp (|n| z+i n x / \kappa), \\
& v_{2}(x, z)=\sum_{\substack{n=-\infty \\
n \neq 0}}^{+\infty} c_{n} \varphi(z) \exp (|n| z=i n x / \kappa),
\end{aligned}
$$

for $-1 \leq z \leq 0$ and $0 \leq x \leq 2 \pi \kappa$, where $\varphi(z)=1$ for $z$ near zero and $\varphi(z)=0$ for $z$ near -1 with $\varphi \in C^{\infty}([-1,0])$. Thus $V \in H_{p}^{1}(\Omega)$ and $\nabla \cdot V=0$ with

$$
v_{2}(x, 0)=h(x)-\frac{1}{2 \pi \kappa} \int_{0}^{2 \pi \kappa} h(s) d s \quad \text { and } \quad\|V\|_{H_{p^{1}}^{1}(\Omega)} \leq C\|h(x)\|_{H_{p}^{1 / 2}(\Gamma)} .
$$

Now let $h(x)=\eta_{n_{k}}-\eta_{m_{k}}$. Since $\eta_{n_{k}} \in H_{p}^{1}(\Gamma), h(x) \in H_{p}^{1}(\Gamma)$ and $V_{k}(x, z)$ in (61) belong to $H_{p}^{3 / 2}(\Omega)$. By the Trace Theorem, $\left.v_{2}\right|_{\Gamma} \in H_{p}^{1}(\Gamma)$ and $\left.v_{2 x}\right|_{\Gamma}$ is well defined and equals $\left(\eta_{n_{k}}-\eta_{m_{k}}\right)_{x}$ on $\Gamma$. However, on the right-hand side of $(60), U_{m_{k}}, U_{n_{k}}$ are bounded in $H_{p}^{1}(\Omega), \bar{G}_{n}$ is bounded in $H$ and the highest derivative of $V_{k}$ is the first-order derivative, which is uniformly bounded by $\left\|\eta_{n_{k}}-\eta_{m_{k}}\right\|_{H_{p}^{1 / 2}(\Gamma)}$. Since $\eta_{n_{k}}$ is convergent in $H_{p}^{1 / 2}(\Gamma)$, $V_{k}$ goes to zero in $H_{p}^{1}(\Omega)$ as $n_{k}, m_{k} \rightarrow+\infty$. By using the Hölder inequality to the terms of the right-hand side of $(60)$, we have that $\int_{\Gamma}\left|\left(\eta_{n_{k}}-\eta_{m_{k}}\right)_{x}\right|^{2} d \Gamma$ does to zero as $n_{k}, m_{k} \rightarrow+\infty$. Thus $\bar{U}_{n_{k}}=\left(U_{n_{k}}, \eta_{n_{k}}\right)^{\mathrm{T}}$ is convergent in $H$. Therefore $\mathcal{B}$ is compact.

From Lemma 2, we see that the spectrum of $\mathcal{B}$ consists of discrete eigenvalues and zero only, and the only possible limit point is zero. By the relation between $\mathcal{A}$ and $\mathcal{B}$, we have 
ThEOREM 1. The spectrum of $\mathcal{A}$ consists of discrete eigenvalues only and lies on the left-hand side of the line $\operatorname{Re} \lambda=\lambda_{0}$.

In the following, we shall show that $\mathcal{A}$ is an infinitesimal generator of an analytic semigroup in $H$. Let $\operatorname{Re} \lambda \geq \lambda_{0}$. Then $(\lambda I-\mathcal{A})^{-1}$ exists. For $\bar{G} \in H,(\lambda I-\mathcal{A})^{-1} \bar{G}=\bar{U}$ satisfies (56) with $\lambda \eta=\left.u_{2}\right|_{\Gamma}+g_{0}$. By choosing $V=U$, we have

$$
\begin{array}{r}
\int_{\Omega}(\lambda U+B U) \cdot U^{*} d \Omega+\langle U, U\rangle+\int_{\Gamma}\left(-\eta u_{1}^{*} \sin \theta+\lambda|\eta|^{2} \cos \theta+\lambda \beta\left|\eta_{x}\right|^{2}\right) d \Gamma \\
=(P G, U)_{L^{2}(\Omega)}+\int_{\Gamma}\left(\eta g_{0} \cos \theta+\beta \eta_{x} g_{0 x}\right) d \Gamma .
\end{array}
$$

By using the Trace Theorem and taking $\lambda_{0}$ large, the real part of (63) becomes

$$
\begin{aligned}
\operatorname{Re}(\lambda & \left.-\lambda_{0}\right)\left(\int_{\Omega}|U|^{2} d \Omega+\int_{\Gamma}\left(\cos \theta|\eta|^{2}+\beta\left|\eta_{x}\right|^{2}\right) d \Gamma\right) \\
& +(1 / 4)\left(\langle U, U\rangle+\|U\|_{L_{p}^{2}(\Omega)}^{2}+\|\eta\|_{H_{p}^{1}(\Gamma)}^{2}\right) \leq\|\bar{G}\|_{H} .
\end{aligned}
$$

Thus

$$
\|\bar{U}\|_{H} \leq\left(\operatorname{Re}\left(\lambda-\lambda_{0}\right)\right)^{-1}\|\bar{G}\|_{H} \quad \text { and } \quad\|U\|_{H_{p}^{1}(\Omega)}+\|\eta\|_{H_{p}^{1}(\Gamma)} \leq C\|\bar{G}\|_{H},
$$

which implies that $\mathcal{A}$ is an infinitesimal generator of a $C_{0}$-semigroup since it is clear that $\mathcal{A}$ is closed and $\mathcal{D}(\mathcal{A})$ is dense in $H$. Also, the imaginary part of (63) takes the form

$$
\begin{aligned}
(\operatorname{Im} \lambda) & \left(\int_{\Omega}|U|^{2} d \Omega+\int_{\Gamma}\left(|\eta|^{2} \cos \theta+\beta\left|\eta_{x}\right|^{2}\right) d \Gamma\right) \\
=- & \operatorname{Im}\left(\int_{\Omega} B U \cdot U^{*} d \Omega-\int_{\Gamma} \eta u_{1}^{*} \sin \theta d \Gamma\right) \\
& \quad+\operatorname{Im}\left((P G, U)_{L_{p}^{2}(\Omega)}+\int_{\Gamma}\left(\eta g_{0} \cos \theta+\beta \eta_{x} g_{0 x}\right) d \Gamma\right) .
\end{aligned}
$$

However, the right-hand side of $(66)$ is less than

$$
C\left(\langle U, U\rangle+\|U\|_{L_{p}^{2}(\Omega)}^{2}+\|\eta\|_{H_{p}^{1}(\Gamma)}^{2}+\|G\|_{L_{p}^{2}(\Omega)}^{2}+\left\|g_{0}\right\|_{H_{p}^{1}(\Gamma)}\right),
$$

for some $C>0$. By (64), for $\operatorname{Re} \lambda \geq \lambda_{0}$ (66) becomes

$$
\|\bar{U}\|_{H} \leq \frac{C}{|\operatorname{Im} \lambda|}\|\bar{G}\|_{H}
$$

where $C$ is a constant independent of $\lambda$. Therefore $\mathcal{A}$ is an infinitesimal generator of an analytic semigroup $T(t)$. For $\operatorname{Re} \lambda \geq \lambda_{0}$, (49) and (50) can be solved if we use the generalized solutions in $H$ for $\operatorname{Re} \lambda \geq \lambda_{0}$. By using a smoothing process similar to elliptic equations, we can have

TheOREm 2. Assume that $\operatorname{Re} \lambda \geq \lambda_{0}$ in (53) and (54). If $G \in H_{p}^{r}(\Omega)$ and $g_{0} \in H_{p}^{r+5 / 2}(\Gamma)$ with $r \geq 0$, then (53) and (54) have a unique solution $\bar{U}=(U, \eta)^{\mathrm{T}}$ with $U=P L^{2}(\Omega)$ satisfying

$$
\begin{aligned}
& \|U\|_{H_{p}^{r+2}(\Omega)}+|\lambda|^{(r+2) / 2}\|U\|_{L_{p}^{2}(\Omega)}+\|\eta\|_{H_{p}^{r+5 / 2}(\mathrm{I})}+|\lambda|^{(r+5 / 2) / 2}\|\eta\|_{L_{p}^{2}(\mathrm{\Gamma})} \\
& \quad \leq C\left(\|P G\|_{H_{p}^{r}(\Omega)}+|\lambda|^{r / 2}\|P G\|_{L_{p}^{2}(\Omega)}+\left\|g_{0}\right\|_{H_{p}^{r+5 / 2}(\mathrm{I})}+|\lambda|^{(r+5 / 2) / 2}\left\|g_{0}\right\|_{L_{p}^{2}(\Gamma)}\right) .
\end{aligned}
$$

Since an analogous proof can be found in the proof of Theorem 1 in [1], we omit the proof here. 
4. Existence of solutions. In this section, we consider the modified linear problem of $(34)$ to $(39)$ : in $\Omega$,

$$
\begin{gathered}
\nabla \cdot U=0 \\
U_{t}+\mu U+\widetilde{U}_{0} \cdot \nabla U+U \cdot \nabla U_{0}+\nabla p-R^{-1} \nabla^{2} U=G(x, z, t)
\end{gathered}
$$

at $z=0$,

$$
\begin{aligned}
\eta_{t}+\mu \eta-u_{2} & =0, \\
R^{-1}\left(u_{1 z}+u_{2 x}\right)-\eta \sin \theta & =g_{1}(x, t), \\
p-(2 / R) u_{2 z}+\beta \eta_{x x}-\eta \cos \theta & =g_{2}(x, t)
\end{aligned}
$$

at $z=-1$,

$$
U=0
$$

at $t=0$,

$$
U=U^{0}, \quad \eta=\eta^{0}
$$

where $U^{0}, \eta^{0}$ satisfy the compatibility conditions for (67) to (72) and $\mu$ is a fixed real number, $G(x, z, t) \in K^{r}\left(\Omega \times \mathbf{R}^{+}\right), g_{1}(x, t)$ and $g_{2}(x, t) \in K^{r+1 / 2}\left(\Gamma \times \mathbf{R}^{+}\right), U^{0} \in H_{p}^{r+3 / 2}(\Omega)$, and $\eta^{0} \in H_{p}^{r+2}(\Gamma)$.

First we can construct two functions $U_{1}(x, z, t) \in K^{r+2}\left(\Omega \times \mathbf{R}^{+}\right), p_{1}(x, z, t) \in$ $K^{r+1}\left(\Omega \times \mathbf{R}^{+}\right)$, and $\eta_{1}(x, t) \in K^{r+5 / 2}\left(\Gamma \times \mathbf{R}^{+}\right)$such that $\nabla \cdot U_{1}=0,\left.U_{1}\right|_{B}=0,\left.u_{12}\right|_{\Gamma}=0$ for all $t \in \mathbf{R}^{+}$and $U_{1}(x, z, 0)=U^{0}$ and $\eta_{1}(x, 0)=\eta^{0}$ for $1<r<3 / 2$ with

$$
\begin{aligned}
& \left\|U_{1}\right\|_{K^{r+2}\left(\Omega \times \mathbf{R}^{+}\right)}+\left\|\eta_{1}\right\|_{K^{r+5 / 2}\left(\Gamma \times \mathbf{R}^{+}\right)}+\left\|p_{1}\right\|_{K^{r-1}\left(\Omega \times \mathbf{R}^{+}\right)} \\
& \leq C\left(\left\|U^{0}\right\|_{H_{p}^{r+3 / 2}(\Omega)}+\left\|\eta^{0}\right\|_{K_{p}^{r+2}(\Gamma)}\right)
\end{aligned}
$$

and the following conditions are satisfied. If we let

$$
U=U_{1}+V_{1}, \quad \eta=\eta_{1}+\zeta_{1}, \quad p=p_{1}+q_{1}
$$

so that $(67)$ to $(73)$ become

$$
\begin{gathered}
\nabla \cdot V_{1}=0 \\
V_{1 t}+\mu V_{1}+\widetilde{U}_{0} \cdot \nabla V_{1}+V_{1} \cdot \nabla U_{0}+\nabla q_{1}-R^{-1} \nabla^{2} V_{1}=G_{1}(x, z, t),
\end{gathered}
$$

then at $z=0$,

$$
\begin{aligned}
\zeta_{1 t}+\mu \zeta_{1}-v_{12} & =0 \\
R^{-1}\left(\left(v_{11}\right)_{z}+\left(v_{12}\right)_{x}\right)-\zeta_{1} \sin \theta & =g_{11}(x, t), \\
q_{1}-(2 / R)\left(v_{12}\right)_{z}+\beta \zeta_{1 x x}-\zeta_{1} \cos \theta & =g_{12}(x, t)
\end{aligned}
$$

at $z=-1$,

$$
V_{1}=0 ;
$$


and at $t=0$,

$$
V_{1}=0, \quad \zeta_{1}=0,
$$

where $V_{1}=\left(v_{11}, v_{12}\right)$, then $G_{1}(x, z, t) \in K_{0}^{r}\left(\Omega \times \mathbf{R}^{+}\right)$and $g_{11}(x, t), g_{12}(x, t) \in$ $K_{0}^{r+1 / 2}\left(\Gamma \times \mathbf{R}^{+}\right)$. Such construction can be done in a fashion similar to Lemma 6.1 in [1] by using the compatibility conditions and choosing appropriate $p_{1} \in K^{r+1}\left(\Omega \times \mathbf{R}^{+}\right)$. Next we further reduce (76) to (82) to equations with $g_{11}=g_{12}=0$. Let $Z(x, z, t)$ be $\left(w_{z},-w_{x}\right)$ where $w(x, 0, t)=w_{z}(x, 0, t)=0, w_{z z}(x, 0, t)=R g_{11}(x, t)$ with $w \equiv 0$ near $z=-1$ and $w$ is periodic in $x$. Such $w(x, z, t)$ always exists and $Z \in K_{0}^{r+2}\left(\Omega \times \mathbf{R}^{+}\right)$with $\|Z\|_{K_{0}^{r+2}\left(\Omega \times \mathbf{R}^{+}\right)} \leq C\left\|g_{11}\right\|_{K_{0}^{r+1 / 2}\left(\Gamma \times \mathbf{R}^{+}\right)}$. Also let $p_{0}(x, z, t)$ satisfy

$$
\nabla^{2} p_{0}(x, z, t)=0 \text { in } \Omega, \quad p_{0 z}=0 \text { on } B, \quad p_{0}=g_{12}(x, t) \text { on } \Gamma \text {. }
$$

From the theory of elliptic equations, we know that $p_{0}$ can always be found and

$$
\left\|p_{0}(x, z, t)\right\|_{K_{0}^{r+1}(\Omega \times \mathbf{R})} \leq C\left\|g_{12}\right\|_{K_{0}^{r+1 / 2}(\Gamma \times \mathbf{R})} .
$$

Let

$$
V=V_{1}-Z, \quad q=q_{1}-p_{0}, \quad \zeta=\zeta_{1} .
$$

Then $V, q$, and $\zeta$ satisfy

$$
\begin{aligned}
& \nabla \cdot V=0 \\
& V_{t}+\mu V+\widetilde{U}_{0} \cdot \nabla V+V \cdot \nabla U_{0}+\nabla q-R^{-1} \nabla^{2} V \\
& =G_{1}(x, y, t)-Z_{t}-\mu Z-\widetilde{U}_{0} \cdot \nabla Z-Z \cdot \nabla U_{0}-\nabla p_{0}+R^{-1} \nabla^{2} Z \\
& =G_{2}(x, z, t) ;
\end{aligned}
$$

at $z=0$,

$$
\begin{aligned}
\zeta_{t}+\mu \zeta-v_{2} & =0 \\
R^{-1}\left(v_{1 z}+v_{2 x}\right)-\zeta \sin \theta & =0 \\
q-(2 / R) v_{2 z}+\beta \zeta_{x x}-\zeta \cos \theta & =0
\end{aligned}
$$

at $z=-1$,

$$
V=0
$$

at $t=0$,

$$
V=0, \quad \zeta=0,
$$

where $G_{2} \in K_{0}^{r}\left(\Omega \times \mathbf{R}^{+}\right)$satisfies

$$
\begin{aligned}
& \left\|G_{2}(x, z, t)\right\|_{K_{0}^{r}\left(\Omega \times \mathbf{R}^{+}\right)} \\
& \quad \leq C\left(\left\|G_{1}(x, z, t)\right\|_{K_{0}^{r}\left(\Omega \times \mathbf{R}^{+}\right)}+\left\|g_{11}(x, t)\right\|_{K_{0}^{r+1 / 2}\left(\Gamma \times \mathbf{R}^{+}\right)}+\left\|g_{12}(x, t)\right\|_{K_{0}^{r+1 / 2}\left(\Gamma \times \mathbf{R}^{+}\right)}\right) \\
& \quad \leq C\left(\|G(x, z, t)\|_{K^{r}\left(\Omega \times \mathbf{R}^{+}\right)}+\left\|g_{1}(x, t)\right\|_{K^{r+1 / 2}\left(\Gamma \times \mathbf{R}^{+}\right)}\right. \\
& \left.\quad+\left\|g_{2}(x, t)\right\|_{K^{r+1 / 2}\left(\Gamma \times \mathbf{R}^{+}\right)}+\left(\left\|U^{0}\right\|_{H_{p}^{r+3 / 2}(\Omega)}+\left\|\eta^{0}\right\|_{H_{p}^{r+2}(\Gamma)}\right)^{2}\right),
\end{aligned}
$$


since the linear terms for $U^{0}, \eta^{0}$ are canceled by the compatibility conditions on $U^{0}, \eta^{0}$. After applying the projection $P$ defined in Sec. 2 to (85), equations (84) to (90) can be rewritten into an operator form:

$$
\begin{gathered}
\left(\begin{array}{l}
V \\
\zeta
\end{array}\right)_{t}+(\mu I-\mathcal{A})\left(\begin{array}{l}
V \\
\zeta
\end{array}\right)=\left(\begin{array}{c}
P G_{2} \\
0
\end{array}\right), \\
\left(\begin{array}{l}
V \\
\zeta
\end{array}\right)=\left(\begin{array}{l}
0 \\
0
\end{array}\right) \quad \text { at } t=0 .
\end{gathered}
$$

However, if $\mu=\lambda_{0}$, the spectrum of $\lambda_{0} I-\mathcal{A}$ is strictly on the left side of the plane and the proof of the existence of solutions for (92) and (93) can be obtained from Theorem 2 in a similar way to the proof of Theorem 2 from Theorem 1 in [1]. Thus we have

THEOREM 3. If $\mu=\lambda_{0}$ and $G_{2}(x, z, t) \in K_{0}^{r}\left(\Omega \times \mathbf{R}^{+}\right)$for $r \geq 0$ and not a half-integer, then $(84)$ to $(90)$ have a unique solution $(V, \zeta, q)$, with

$$
\|V\|_{K_{0}^{r+2}\left(\Omega \times \mathbf{R}^{+}\right)}+\|\zeta\|_{K_{0}^{r+5 / 2}\left(\Gamma \times \mathbf{R}^{+}\right)}+\|q\|_{K_{0}^{r+1}\left(\Omega \times \mathbf{R}^{+}\right)} \leq C\left\|G_{2}\right\|_{K_{0}^{r}\left(\Omega \times \mathbf{R}^{+}\right)} .
$$

Now let us consider (67) to (73) with $G, g_{1}, g_{2}$ substituted by

$$
F(U, \eta, p), \quad f_{1}(U, \eta, p), \quad f_{2}(U, \eta, p)
$$

in (35) to (37). By using the algebraic properties of the space $K^{r}\left(\Omega \times \mathbf{R}^{+}\right)$or $K^{r}\left(\Gamma \times \mathbf{R}^{+}\right)$ and the properties for composite functions in $K^{r}\left(\Gamma \times \mathbf{R}^{+}\right.$) (see Lemmas 5.1 and 5.2 in [1]), we have that if $U \in K^{r+2}\left(\Omega \times \mathbf{R}^{+}\right), p \in K^{r+1}\left(\Omega \times \mathbf{R}^{+}\right), \eta \in K^{r+5 / 2}\left(\Gamma \times \mathbf{R}^{+}\right)$, then $F \in K^{r}\left(\Omega \times \mathbf{R}^{+}\right)$and $f_{1}, f_{2} \in K^{r+1 / 2}\left(\Omega \times \mathbf{R}^{+}\right)$for $r>1$ with nonlinearity larger than or equal to the order of two with respect to $U, \eta, p$. Then by using (75), (83), (91), Theorem 3 , and the contraction mapping theorem, we can prove the existence of a unique solution for (67) to (73) with $G, g_{1}, g_{2}$ replaced by $F, f_{1}, f_{2}$ in (35) to (37), $\mu=\lambda_{0}$, and small initial conditions. Also we note that the solution for $t>0$ is smoother than the initial conditions. Actually the solution can have derivatives up to any finite order. Now we state the existence theorem.

Theorem 4. If $r, k$, and $T$ are given with $1<r<3 / 2, T>0$, and $k$ a positive integer, then there is a $\delta_{0}>0$ such that for initial values $\eta^{0}, U^{0}$ satisfying

$$
\delta=\left\|\eta^{0}\right\|_{H_{p}^{r+2}(\mathrm{I})}+\left\|U^{(0}\right\|_{H_{p}^{r+3 / 2}(\Omega)} \leq \delta_{0},
$$

and the compatibility conditions for (67) to (72) with $G, g_{1}, g_{2}$ replaced by $F, f_{1}, f_{2}$ in (35) to (37) and $\mu=\lambda_{0}$, there exists a unique solution $(U, \eta, p)$ of $(67)$ to (72) with the following properties:

(1) $U \in K^{r+2}\left(\Omega \times \mathbf{R}^{+}\right), \eta \in K^{r+5 / 2}\left(\Gamma \times \mathbf{R}^{+}\right)$, and $p \in K^{r+1}\left(\Omega \times \mathbf{R}^{+}\right)$with

$$
\|U\|_{K^{r+2}\left(\Omega 2 \times \mathbf{R}^{+}\right)}+\|\eta\|_{K^{r+5 / 2}\left(\Gamma \times \mathbf{R}^{+}\right)}+\|p\|_{K^{r+1}\left(\Omega 2 \times \mathbf{R}^{+}\right)} \leq C \delta,
$$

where $C$ is a constant independent of $\delta$. 
(2) The solution satisfies $\eta \in K^{r+k+5 / 2}(\Gamma \times(T, \infty)), u \in K^{r+k+2}(\Omega \times(T,+\infty))$, $p \in K^{r+k+1}(\Omega \times(T,+\infty))$, and the sum of these corresponding norms is less than $C_{1} \delta$ where $C_{1}$ is a constant and may depend upon $T$.

Since the proof of this theorem is similar to Sec. 5 in [1], we shall omit it here. We note that the situation considered here is simpler than the one in [1] since the spectrum of the operator in [1] contains zero while we have no such difficulty here. Also the domain is bounded here, which makes the proof easier to carry over by using Fourier series rather than Fourier integrals in [1].

REMARK.

(1). The condition $\mu=\lambda_{0}$ is not necessary. This condition assures that $\mu I-\mathcal{A}$ has its spectrum lying on the left half-plane. If the spectrum of $\mu I-\mathcal{A}$ is in the left half-plane for smaller $\mu$, Theorem 4 still holds for such $\mu$. For $\mu=0,(67)$ to (72) become (34) to (39).

(2). If we are only interested in the existence of solutions of (34) to (39) up to a fixed finite time $T_{0}$, then we can use the transformation $U=e^{\mu t} W, \eta=e^{\mu t} \xi, p=e^{\mu t} p_{3}$, multiply a smooth function $\varphi(t)$ with $\varphi(t) \equiv 1$ for $|t| \leq 2 T_{0}$ and $\varphi(t) \equiv 0$ for $|t| \geq 3 T_{0}+10$, and transform (34) to (39) into (67) to (72), which implies the existence of solutions in $0<t \leq T_{0}$. Therefore for small initial data, the existence of solutions of (34) to (39) up to a fixed finite time is a consequence of Theorem 4 .

5. Stability of the motion of the fluid. In this section, we shall show that the solutions of (34) to (39) with small initial conditions are stable under some conditions on the spectrum of $\mathcal{A}$. The assumption is that $\mathcal{A}$ has no eigenvalue $\mu$ with $\operatorname{Re} \mu \geq 0$. This can be verified easily by numerical computation, and it has been done in [5] using normal mode analysis and finding the neutral curves for the linear operator $\mathcal{A}$. We note here that we need to use (22) to transform the results in [5] into the conditions on the spectrum of $\mathcal{A}$. Since the spectrum $\sigma(\mathcal{A})$ is closed and $\mathcal{A}$ is an infinitesimal generator of an analytic semigroup, we can find two positive constants $\omega, \delta$ such that

$$
\sigma(\mathcal{A}) \in \Delta_{\delta}=\{z|| \arg (z-2 \omega) \mid>\delta+\pi / 2\} .
$$

Now we use the following change of variables in (34) to (39):

$$
U=e^{-\omega t} U^{(1)}, \quad \eta=e^{-\omega t} \eta^{(1)}, \quad p=e^{-\omega t} p^{(1)},
$$

with some initial conditions. (34) to (39) then become (67) to (73) with $\mu=-\omega$ and

$$
\left[\begin{array}{c}
G(x, z, t) \\
g_{1}(x, t) \\
g_{2}(x, t)
\end{array}\right]=e^{\omega t}\left[\begin{array}{c}
F \\
f_{1} \\
f_{2}
\end{array}\right]\left(e^{-\omega t} U^{(1)}, e^{-\omega t} \eta^{(1)}, e^{-\omega t} p^{(1)}\right)
$$

where $U, \eta, p$ are replaced by $U^{(1)}, \eta^{(1)}, p^{(1)}$, respectively. First, we need a theorem similar to Theorem 2 in this case:

THEOREM 5. If $G(x, z) \in H_{p}^{r}(\Omega)$ and $g_{0}(x) \in H_{p}^{r+5 / 2}(\Gamma)$ with $r \geq 0$, then for $\operatorname{Re} \lambda \geq 0$, the equation

$$
(\lambda I-(\omega I-\mathcal{A})) \bar{U}=\left(\begin{array}{c}
P G \\
g_{0}
\end{array}\right)=\bar{G}
$$


has a unique solution $\bar{U}=(U, \eta)^{\mathrm{T}}$ and the solution satisfies

$$
\begin{aligned}
& \|U\|_{H_{p}^{r+2}(\Omega)}+|\lambda|^{(r+2) / 2}\|U\|_{L_{p}^{2}(\Omega)}+\|\eta\|_{H_{p}^{r+5 / 2}(\Gamma)}+|\lambda|^{(r+5 / 2) / 2}\|\eta\|_{L_{p}^{2}(\Gamma)} \\
& \quad \leq C\left(\|P G\|_{H_{p}^{r}(\Omega)}+|\lambda|^{r / 2}\|P G\|_{L_{p}^{2}(\Omega)}+\left\|g_{0}\right\|_{H_{p}^{r+5 / 2}(\Gamma)}+|\lambda|^{(r+5 / 2) / 2}\left\|g_{0}\right\|_{L_{p}^{2}(\Gamma)}\right) .
\end{aligned}
$$

Proof. We just show very briefly how to obtain the estimates since the majority of the proof is the same as the one for Theorem 2 and also can be found in [1]. Because $\lambda I-(\omega I+\mathcal{A})$ is invertible for $\operatorname{Re} \lambda \geq 0$ and $\omega I+\mathcal{A}$ is an infinitesimal generator of an analytic semigroup, we have

$$
\|\bar{U}\|_{H}=\left\|(\lambda I-(\omega I+\mathcal{A}))^{-1} \bar{G}\right\|_{H} \leq(C /(1+|\lambda|))\|\bar{G}\|_{H}
$$

Therefore, $U$ in $L^{2}(\Omega)$ and $\eta$ in $H^{1}(\Gamma)$ are bounded by $\|\bar{G}\|_{H}$. If $|\lambda|$ is large, by (96) the proof of Theorem 2 can be carried over and the theorem can be proved. Thus we only have to prove the case when $|\lambda|$ is bounded. From (56), we let $V=U$ and use (96) and $\left.u_{2}\right|_{\Gamma} \in H_{p}^{1}(\Gamma)$ to have a bounded estimate of $\|U\|_{H_{p}^{1}(\Omega)}$. Denote

$$
\Lambda_{n}^{m} f(x, z)=\sum_{k=-n}^{n}\left(\frac{i k}{2 \pi \kappa}\right)^{m} \exp \left(\frac{i k}{2 \pi \kappa}\right)(2 \pi \kappa)^{-1} \int_{0}^{2 \pi \kappa} f(x, z) \exp \left(\frac{-i k}{2 \pi \kappa}\right) d x
$$

Then construct a function $V=\left(v_{1}(x, z), v_{2}(x, z)\right)$ with $\nabla \cdot V=0$ and $\left.v_{2}\right|_{z=0}=\Lambda_{n}^{1} \eta$ and $V \equiv 0$ near $z=-1$. Such a function has been obtained in (61) and (62). Also $V \in H_{p}^{1}(\Omega)$ if $\Lambda_{n}^{1} \eta \in H_{p}^{1 / 2}(\Gamma)$ and $\|V\|_{H_{p}^{1}(\Omega)} \leq C\left\|\Lambda_{n}^{1} \eta\right\|_{H_{p}^{1 / 2}(\Gamma)}$. We substitute $V$ into (56) to obtain

$$
\begin{aligned}
\int_{\Gamma} \beta\left|\left(\Lambda_{n}^{1 / 2} \eta\right)_{x}\right|^{2} d \Gamma & =\left|\int_{\Gamma} \beta \eta_{x}\left(\Lambda_{n}^{1} \eta\right)_{x}^{*} d \Gamma\right| \\
& \leq C_{1}\left(\|U\|_{H_{p}^{1}(\Omega)}\|V\|_{H_{p}^{1}(\Omega)}+\|U\|_{H_{p}^{1}(\Omega)}^{2}+\|\eta\|_{H_{p}^{1}(\Gamma)}^{2}+\|\bar{G}\|_{H}^{2}\right) \\
& \leq(\beta / 2)\left\|\Lambda_{n}^{1} \eta\right\|_{H_{p}^{1 / 2}(\Gamma)}^{2}+C_{2}\|\bar{G}\|_{H}^{2} .
\end{aligned}
$$

However, since $\Lambda_{n}^{1 / 2} \eta$ has only finite terms,

$$
\left\|\Lambda_{n}^{1} \eta\right\|_{H_{p}^{1 / 2}(\Gamma)}^{2} \leq\left\|\left(\Lambda_{n}^{1 / 2} \eta\right)_{x}\right\|_{L_{p}^{2}(\Omega)}^{2}+C_{3}\|\eta\|_{H_{p}^{1}(\Gamma)}^{2}
$$

Thus we have

$$
\left\|\left(\Lambda_{n}^{1 / 2} \eta\right)_{x}\right\|_{L_{p}^{2}(\Gamma)}^{2} \leq C_{4}\|\bar{G}\|_{H}^{2}
$$

Let $n \rightarrow+\infty$ and obtain

$$
\left\|\eta_{x}\right\|_{H_{p}^{1 / 2}(\Gamma)}^{2} \leq C_{5}\|\bar{G}\|_{H}^{2}
$$

which implies that $\eta \in H_{p}^{3 / 2}(\Gamma)$ and $\|\eta\|_{H_{p}^{3 / 2}(\Gamma)}^{2} \leq C_{5}\|\bar{G}\|_{H}^{2}$. Then by (56) again, we choose $V=\Lambda_{n}^{1}(U)$ and by the estimates on $\eta$ and using the same procedure in the proof of Theorem 1 in $[1]$, we have $U \in H_{p}^{3 / 2}(\Omega)$ and $\|U\|_{H_{p}^{3 / 2}(\Omega)} \leq C\left(\|\bar{G}\|_{H}+\left\|g_{0}\right\|_{H_{p}^{3 / 2}(\Gamma)}\right)$. By using the same argument to obtain $\eta \in H_{p}^{3 / 2}(\Gamma)$, we have $\eta \in H_{p}^{2}(\Gamma)$ and then 
$U \in H_{p}^{2}(\Omega)$. Finally $\eta \in H_{p}^{5 / 2}(\Gamma)$ with the estimate stated in the theorem for $r=0$. The case for $r>0$ is a simple extension to the case $r=0$ by using (96). Thus we prove the theorem.

After we have Theorem 5 and notice that $F, f_{1}, f_{2}$ contain all nonlinear terms so that at least one factor of $\exp (-\omega t)$ appears in $G$ or $g_{1}$ or $g_{2}$, the existence of solutions of (67) to (73) with $\mu=-\omega$ and $G, g_{1}, g_{2}$ defined in (95) with small initial conditions can be established in a way similar to the proof of Theorem 4 from Theorem 2. Therefore, we also have Theorem 4 for the solutions of (67) to (73) with $\mu=-\omega$ and $G, g_{1}, g_{2}$ in (95). By (2) of Theorem 4, we choose $k$ large enough such that for $t \in[T,+\infty)$ with a fixed integer $k_{1}>0, \delta$ small and $T>0, \eta^{(1)}(\cdot, t) \in H_{p}^{r+k_{1}+5 / 2}(\Gamma), U^{(1)}(\cdot, t) \in H_{p}^{r+k_{1}+2}(\Omega)$, $p^{(1)}(\cdot, t) \in H_{p}^{r+k_{1}+1}(\Omega)$, and

$$
\left\|\eta^{(1)}\right\|_{H_{p}^{r+k_{1}+5 / 2}(\Gamma)}+\left\|U^{(1)}\right\|_{H_{p}^{r+k_{1}+2}(\Omega)}+\left\|p^{(1)}\right\|_{H_{p}^{r+k_{1}+1}(\Omega)} \leq C \delta,
$$

if $\left\|\eta^{0}\right\|_{H_{p}^{r+2}(\Gamma)}+\left\|U^{0}\right\|_{H_{p}^{r+(3 / 2)}(\Omega)}=\delta \leq \delta_{0}$ with $\eta^{0}$ and $U^{0}$ satisfying the compatibility condition and $1<r<3 / 2$. Then by the definition of $\eta^{(1)}, U^{(1)}, p^{(1)}$, we have

$$
\|\eta\|_{H_{p}^{r+k_{1}+5 / 2}(\Gamma)}+\|U\|_{H_{p}^{r+k_{1}+2}(\Omega)}+\|p\|_{H_{p}^{r+k_{1}+1}(\Omega)} \leq C \delta \exp (-\omega t),
$$

for $t \in[T,+\infty)$. When $t \rightarrow+\infty,\|\eta\|_{H_{p}^{r+k_{1}+5 / 2}(\Gamma)}+\|U\|_{H_{p}^{r+k_{1}+2}(\Omega)}+\|p\|_{H_{p}^{r+k_{1}+1}(\Omega)}$ goes to zero exponentially. If we transform (34) to (39) back to equations (1) to (5), we can see that all the transformations are invertible, (1) to (5) have solutions if the initial conditions satisfy the compatibility conditions and are very close to the steady state (14), and the solutions approach the steady state exponentially. Thus we have the following stability theorem.

Theorem 6. Assume that

$$
\Omega^{*}=\Gamma \times\left(-1, \zeta^{*}\right), \quad U_{0}^{*}=\left((R \sin \theta / 2)\left(1-\left(z^{*} / h^{*}\right)^{2}\right), 0\right), \quad p_{0}^{*}=-\left(z^{*} /\left(h^{*} \cos \theta\right)\right),
$$

and the initial conditions $\zeta_{0}^{*}, \underline{q}_{0}^{*}$ of $\zeta^{*}, \underline{q}^{*}$ satisfy the compatibility conditions. Define $H_{p}^{r}\left(\Omega^{*}\right)$ and $K^{r}\left(\Omega^{*} \times(0, T)\right)$ in a way similar to $H_{p}^{r}(\Omega)$ and $K^{r}(\Omega \times(0, T))$. Let $r_{1}>1$ be any fixed integer and $1<r<3 / 2$. If $\mathcal{A}$ in (51) has only eigenvalues with negative real part, then there exists a $\delta_{0}>0$ and $\omega>0$ such that for $\left\|\zeta_{0}^{*}\right\|_{H_{p}^{r+2}(\Gamma)}+\| \underline{q}_{0}^{*}-$ $U_{0}^{*} \|_{H_{p}^{r+3 / 2}\left(\Omega^{*}\right)}=\delta \leq \delta_{0}$ the equations (1) to (7) have a solution $\left(\zeta^{*}, \underline{q}^{*}, p^{*}\right)$ with

$$
\left\|\zeta^{*}\right\|_{K^{r+5 / 2}(\Gamma \times(0,+\infty))}+\left\|\underline{q}^{*}-U_{0}^{*}\right\|_{K^{r+2}\left(\Omega^{*} \times(0,+\infty)\right)}+\left\|p^{*}-p_{0}^{*}\right\|_{K^{r+1}\left(\Omega^{*} \times(0,+\infty)\right)} \leq C \delta,
$$

and for $t$ large, $\zeta^{*}(\cdot, t) \in H_{p}^{r_{1}+1 / 2}(\Gamma), \underline{q}^{*}(\cdot, t) \in H_{p}^{r_{1}}\left(\Omega^{*}\right), p^{*}(\cdot, t) \in H_{p}^{r_{1}-1}\left(\Omega^{*}\right)$, and

$$
\left\|\zeta^{*}(\cdot, t)\right\|_{H_{p}^{r_{1}+1 / 2}(\Gamma)}+\left\|\underline{q}^{*}(\cdot, t)-U_{0}^{*}\right\|_{H_{p}^{r_{1}}\left(\Omega^{*}\right)}+\left\|p(\cdot, t)-p_{0}^{*}\right\|_{H_{p}^{r_{1}-1}\left(\Omega^{*}\right)} \leq C \exp (-\omega t),
$$

where $C$ is a constant independent of $t$ and $\delta$.

Obviously Theorem 6 implies the global existence of solutions for small initial data. 
6. Instability of the motion of the fluid. Here we assume that $\mathcal{A}$ has eigenvalues with $\operatorname{Re} \lambda>0$. Since $\mathcal{A}$ has only discrete eigenvalues and the only limit point of the eigenvalues is $-\infty$, there are only finite eigenvalues with positive real part. Let $\delta_{i}$, $i=1,2,3, \ldots, n$ be the eigenvalues with $\operatorname{Re} \delta_{i}=\max (\operatorname{Re} \sigma(\mathcal{A}))=\delta_{0}$ for $i=1,2, \ldots, n$. Since $\mathcal{A}$ generates an analytic semigroup in $H$, the identity map

$$
I=(2 \pi i)^{-1} \int_{\gamma}(\lambda I-\mathcal{A})^{-1} d \lambda
$$

where $\sigma(\mathcal{A})$ lies on the left-hand side of the curve $\gamma$ which goes from $+\infty e^{i \phi}$ to $+\infty^{-i \phi}$ with $\phi>(\pi / 2)$ in the complex plane. Let $\gamma^{-}$be a curve with real part less than $\delta_{0}-\omega>0$ for some small $\omega>0$ and let $\sigma(\mathcal{A})$ be on the left-hand side of $\gamma^{-}$with distance to $\gamma^{-}$ larger than $\omega$ except $\left\{\delta_{i}\right\}_{i=1}^{n}$. Then define

$$
\begin{gathered}
P_{-}=(2 \pi i)^{-1} \int_{\gamma^{-}}(\lambda-\mathcal{A})^{-1} d \lambda, \quad P_{+}=I-P_{-}, \\
\mathcal{A}=\mathcal{A}_{+}+\mathcal{A}_{-} \quad \text { with } \quad \mathcal{A}_{+}=P_{+} \mathcal{A}, \mathcal{A}_{-}=P_{-} \mathcal{A} .
\end{gathered}
$$

The space $H$ is decomposed into a direct sum of $H_{+}=P_{+}(H)$ and $H_{-}=P_{-}(H)$. The subspaces $H_{+}$and $H_{-}$are invariant relative to $\mathcal{A}$. They contain dense linear spaces $P_{+}(\mathcal{D}(\mathcal{A}))$ and $P_{-}(\mathcal{D}(\mathcal{A}))$, respectively. Note that $P^{+}$is a projection onto a finitedimensional space spanned by the eigenfunctions corresponding to $\delta_{i}, i=1,2, \ldots, n$ and these eigenfunctions are infinitely differentiable by a usual regularity argument.

Let

$$
\left(\begin{array}{c}
U \\
\eta \\
p
\end{array}\right)=\exp \left(\left(\delta_{0}-\omega\right) t\right)\left(\begin{array}{c}
U^{(1)} \\
\eta^{(1)} \\
p^{(1)}
\end{array}\right)
$$

Then (34) to (39) become (67) to (72) with $\mu=\delta_{0}-\omega$, and

$$
\left[\begin{array}{c}
G(x, z, t) \\
g_{1}(x, t) \\
g_{2}(x, t)
\end{array}\right]=e^{-\left(\delta_{0}-\omega\right) t}\left[\begin{array}{c}
F \\
f_{1} \\
f_{2}
\end{array}\right]\left(e^{\left(\delta_{0}-\omega\right) t} U^{(1)}, e^{\left(\delta_{0}-\omega\right) t} \eta^{(1)}, e^{\left(\delta_{1}-\omega\right) t} p^{(1)}\right) .
$$

Let

$$
V_{ \pm}=U_{ \pm}^{(1)}-Z_{ \pm}, \quad \zeta_{ \pm}=\eta_{ \pm}^{(1)}
$$

where $Z$ is constructed in (83) to make $g_{1}, g_{2}$ be zero. If we denote $G$ in (97) by $\widetilde{G}$ after the change of variables using $(98),(67)$ to $(72)$ become

$$
\left(\begin{array}{c}
V_{ \pm} \\
\zeta_{ \pm}
\end{array}\right)_{t}+\left(\left(\delta_{0}-\omega\right) P_{ \pm}-\mathcal{A}_{ \pm}\right)\left(\begin{array}{c}
V_{ \pm} \\
\zeta_{ \pm}
\end{array}\right)=P_{ \pm}\left(\begin{array}{c}
P \widetilde{G} \\
0
\end{array}\right)
$$

However, $\left(\delta_{0}-\omega\right) P_{-}-\mathcal{A}_{-}$is an infinitesimal generator of an analytic semigroup on $H_{-}$and its spectrum $\sigma\left(\left(\delta_{0}-\omega\right) P_{-}-\mathcal{A}_{-}\right)$lies entirely on the left half-plane. Consider

$$
\begin{gathered}
\left(\bar{V}_{-}\right)_{t}+\left(\left(\delta_{0}-\omega\right) I-\mathcal{A}_{-}\right) \bar{V}_{-}=P_{-}\left(\varphi(t)\left(\begin{array}{c}
P \widetilde{G} \\
0
\end{array}\right)\right), \\
\left.\bar{V}\right|_{t=-\infty}=0
\end{gathered}
$$


in the space $H_{-}$where $\bar{V}_{-}=\left(V_{-}, \zeta_{-}\right)^{\mathrm{T}}$ and $\varphi(t) \in C^{\infty}(-\infty,+\infty)$ with $\varphi(t) \equiv 1$ for $t \leq 5, \varphi(t) \equiv 0$ for $t \geq 10$. Since $\left(\delta_{0}-\omega\right) I-\mathcal{A}_{-}$has eigenvalues on the left half-plane, we can have the same estimates as (96) in $H_{-}$. Then by using arguments similar to Theorem $5,\left(\lambda I-\left(\left(\delta_{0}-\omega\right) I-\mathcal{A}_{-}\right)\right) \bar{V}_{-}=P_{-}\left((P \widetilde{G}, 0)^{\mathrm{T}}\right)$ will have solutions for $\operatorname{Re} \lambda \geq 0$ satisfying the inequality in Theorem 5 . Note here that although the scalar component of $(P \widetilde{G}, 0)^{\mathrm{T}}$ is zero, the scalar component of $P_{-}\left((P \widetilde{G}, 0)^{\mathrm{T}}\right)$ may not be zero. However,

$$
\left(\begin{array}{c}
P \widetilde{G} \\
0
\end{array}\right)=P_{-}\left(\begin{array}{c}
P \widetilde{G} \\
0
\end{array}\right)+P_{+}\left(\begin{array}{c}
P \widetilde{G} \\
0
\end{array}\right)
$$

and $P_{+}\left((P \widetilde{G}, 0)^{\mathrm{T}}\right)$ is finite-dimensional and the basis has bounded derivatives up to any finite order. Thus the scalar component of $P_{+}\left((P \widetilde{G}, 0)^{\mathrm{T}}\right)$ is controlled by the $L^{2}$-norm of $P_{-}\left((P \widetilde{G}, 0)^{\mathrm{T}}\right)$, which yields that the derivatives of the scalar component of $P_{-}\left((P \widetilde{G}, 0)^{\mathrm{T}}\right)$ are bounded by the $L^{2}$-norm of $P_{-}\left((P \widetilde{G}, 0)^{\mathrm{T}}\right)$. From the similar inequality in Theorem 5 and the note we just stated, we can obtain results similar to Theorem 4 for (99) if $\left(V_{+}, \zeta_{+}\right)$is small and smooth and satisfies the compatibility condition $\left(V_{+}, \zeta_{+}\right) \rightarrow 0$ as $t \rightarrow-\infty$. We note that the situation here is much easier than the one in Theorem 4 since $\left(V_{+}, \zeta_{+}\right)$is in a finite-dimensional space and the basis is smooth in $(x, z)$, which implies that the conditions on $\left(V_{+}, \zeta_{+}\right)$can be imposed on the coefficients of its linear representation. Also we remark that if $V_{+} \in K^{r+2}(\Omega \times \mathbf{R}), \zeta_{+} \in K^{r+5 / 2}(\Gamma \times \mathbf{R})$, then $V_{-} \in K^{r+2}(\Omega \times \mathbf{R})$ and $\zeta_{-} \in K^{r+5 / 2}(\Gamma \times \mathbf{R})$. Therefore, we have

$$
\left\|V_{-}\right\|_{K^{r+2}(\Omega 2 \times \mathbf{R})}+\left\|\zeta_{-}\right\|_{K^{r+5 / 2}(\Omega 2 \times \mathbf{R})}+\left\|p_{-}\right\|_{K^{r+1}(\Omega \times \mathbf{R})} \leq C \delta
$$

if $\left\|V_{+}\right\|_{K^{r+2}(\Omega \times \mathbf{R})}+\left\|\zeta_{+}\right\|_{K^{r+5 / 2}(\Omega \times \mathbf{R})} \leq \delta$ for small $\delta$, where we have used the fact that $p$ is exclusively determined by $V$ and $\zeta$. Since $\widetilde{G}$ is nonlinear with order at least two, (100) yields the inequality

$$
\left\|V_{-}\right\|_{K^{r+2}(\Omega \times \mathbf{R})}+\left\|\zeta_{-}\right\|_{K^{r+5 / 2}(\Gamma \times \mathbf{R})}+\left\|p_{-}\right\|_{K^{r+1}(\Omega \times \mathbf{R})} \leq C_{1} \delta^{2} .
$$

Therefore $\left(V_{-}, \zeta_{-}, p_{-}\right)$is determined by $\left(V_{+}, \zeta_{+}, p_{+}\right)$. However, $\left(V_{+}, \zeta_{+}\right)$is in a finitedimensional space and the solution of the ordinary differential equation

$$
\left(\begin{array}{c}
V_{+} \\
\zeta_{+}
\end{array}\right)_{t}+\left(\left(\delta_{0}-\omega\right) P_{+}-\mathcal{A}_{+}\right)\left(\begin{array}{c}
V_{+} \\
\zeta_{+}
\end{array}\right)=\left.P_{+}\left(\begin{array}{c}
P \widetilde{G} \\
0
\end{array}\right)\left(\begin{array}{c}
V_{+} \\
\zeta_{+}
\end{array}\right)\right|_{t=0}=\left(\begin{array}{c}
V_{0} \\
\zeta_{0}
\end{array}\right)
$$

can be rewritten as

$$
\left(\begin{array}{c}
V_{+} \\
\zeta_{+}
\end{array}\right)=e^{-\left(\left(\delta_{0}-\omega\right) P_{+}-\mathcal{A}_{+}\right) t}\left(\begin{array}{c}
V_{0} \\
\zeta_{0}
\end{array}\right)+\int_{0}^{t} e^{-\left(\left(\delta_{0}-\omega\right) P_{+}-\mathcal{A}_{+}\right)(t-r)} P_{+}\left(\begin{array}{c}
P \widetilde{G} \\
0
\end{array}\right) d \tau .
$$

The eigenvalues of $\left(\delta_{0}-\omega\right) P_{+}-\mathcal{A}_{-}$are on $\operatorname{Re} \lambda=-\omega<0$. As $t \rightarrow \infty$, the operator $\exp \left(\left(-\left(\delta_{0}-\omega\right) P_{+}-A_{+}\right) t\right)$ tends to infinity. Thus we consider the following modified equation:

$$
\begin{aligned}
&\left(\begin{array}{c}
V_{+} \\
\zeta_{+}
\end{array}\right)=\varphi(t) \exp \left(-\left(\left(\delta_{0}-\omega\right) P_{+}-\mathcal{A}_{+}\right) t\right)\left(\begin{array}{c}
V_{0} \\
\zeta_{0}
\end{array}\right) \\
&+\varphi(t) \int_{0}^{t} \exp \left(-\left(\left(\delta_{0}-\omega\right) P_{+}-\mathcal{A}_{+}\right)(t-\tau)\right) \varphi(\tau) P_{+}\left(\begin{array}{c}
P \widetilde{G} \\
0
\end{array}\right) d \tau
\end{aligned}
$$


where $\varphi(t)$ is defined in (99). (102) is a finite-dimensional integral equation. If $\left(V_{+}, \zeta_{+}\right)^{\mathrm{T}}$ has $(r / 2)$-derivatives with respect to $t$ in the $L^{2}$-norm, $P \widetilde{G}$ has $(r-2) / 2$-derivatives in the $L^{2}$-norm for $r \geq 2$ by using (101). Also $\widetilde{G}$ only consists of nonlinear terms with order at least two. Thus from (101) and routing arguments in nonlinear ordinary differential equations, the right-hand side of (102) is a contraction in a small ball of $H^{r / 2}(\mathbf{R})$ with independent variable $t$ if $\left(V_{0}, \zeta_{0}\right)^{\mathrm{T}}$ is small. By the contraction mapping theorem, for a fixed $r \geq 2$, (102) has a solution in $H^{r / 2}(\mathbf{R})$ for small initial conditions. Next we choose $r$ large such that for a given $r_{1}>0, V_{-}(\cdot, t) \in H_{p}^{r_{1}+2}(\Omega), \zeta_{-}(\cdot, t) \in H_{p}^{r_{1}+5 / 2}(\Gamma)$, $p_{-}(\cdot, t) \in H_{p}^{r_{1}+1}(\Omega)$, and for $t \in \mathbf{R}$,

$$
\begin{aligned}
& \left\|V_{-}(\cdot, t)\right\|_{H_{p}^{r_{1}+2}(\Omega)}+\left\|\zeta_{-}(\cdot, t)\right\|_{H_{p}^{r_{1}+5 / 2}(\Gamma)}+\left\|p_{-}(\cdot, t)\right\|_{H_{p}^{r_{1}+1}(\Omega)} \\
& \quad \leq C_{1}\left(\left\|V_{+}\right\|_{K^{r+2}(\Omega \times \mathbf{R})}+\left\|\zeta_{+}\right\|_{K^{r+5 / 2}(\Gamma \times \mathbf{R})}+\left\|p_{+}\right\|_{K^{r+1}(\Omega \times \mathbf{R})}\right)^{2} \\
& \quad \leq C_{2} \delta^{2}
\end{aligned}
$$

if $\left\|\left(V_{0}, \zeta_{0}\right)\right\|=\delta$, where $C_{1}, C_{2}$ are independent of $\delta$ and $\left\|\left(V_{0}, \zeta_{0}\right)\right\|$ can be any norm for $\left(V_{0}, \zeta_{0}\right)$ since the norms are equivalent in a finite-dimensional space. Thus

$$
\left(\begin{array}{l}
V \\
\zeta
\end{array}\right)=\left(\begin{array}{l}
V_{+} \\
\zeta_{+}
\end{array}\right)+\left(\begin{array}{l}
V_{-} \\
\zeta_{-}
\end{array}\right)
$$

is a classical solution of (67) to (72) with $G=\widetilde{G}$ and $g_{1}=g_{2}=0$ in $t \in(-\infty, 1]$ if $r_{1}$ is chosen in (103). From (98), $U_{ \pm}^{(1)}$ and $\eta_{ \pm}^{(1)}$ have the same properties as $V_{ \pm}$and $\zeta_{ \pm}$since $Z_{ \pm}$only has nonlinear terms of $U_{ \pm}^{(1)}$ and the implicit function theorem can be used to obtain $U_{ \pm}^{(1)}$. Since by definition

$$
\left(\begin{array}{l}
U \\
\eta
\end{array}\right)=\exp \left(\left(\delta_{0}-\omega\right) t\right)\left(\left(\begin{array}{c}
U_{+}^{(1)} \\
\eta_{+}^{(1)}
\end{array}\right)+\left(\begin{array}{c}
U_{-}^{(1)} \\
\eta_{-}^{(1)}
\end{array}\right)\right),
$$

$(U, \eta) \rightarrow 0$ as $t \rightarrow-\infty$ with $U(\cdot, t) \in H_{p}^{r+2}(\Omega)$ and $\eta(\cdot, t) \in H_{p}^{r+5 / 2}(\Gamma)$. However,

$$
\left.\left(\begin{array}{l}
U \\
\eta
\end{array}\right)\right|_{t=0}=\left.\left(\begin{array}{c}
U_{+}^{(1)} \\
\eta_{+}^{(1)}
\end{array}\right)\right|_{t=0}+\left.\left(\begin{array}{c}
U_{-}^{(1)} \\
\eta_{-}^{(1)}
\end{array}\right)\right|_{t=0} .
$$

Then (103) yields

$$
\begin{aligned}
& \|U(\cdot, 0)\|_{H_{p}^{r_{1}+2}(\Omega)}+\|\eta(\cdot, 0)\|_{H_{p}^{r_{1}+5 / 2}(\Gamma)} \\
& \quad \geq\left\|\left(V_{0}, \eta_{0}\right)\right\|-\left(\left\|V_{-}^{(1)}(\cdot, 0)\right\|_{H_{p}^{r_{1}+2}(\Omega)}+\left\|\eta_{-}(\cdot, 0)\right\|_{H_{p}^{r_{1}+5 / 2}(\Gamma)}\right) \\
& \quad \geq \delta-C_{2} \delta^{2}=\delta\left(1-C_{2} \delta\right) .
\end{aligned}
$$

If $\delta>0$ is chosen so small that $1-C_{2} \delta \geq 1 / 2$, then

$$
\|U(\cdot, 0)\|_{H_{p}^{r_{1}+2}(\Omega)}+\eta\|(\cdot, 0)\|_{H_{p}^{r_{1}+5 / 2}(\mathrm{\Gamma})} \geq \delta / 2 \neq 0 .
$$

However, $U$ and $\eta \rightarrow 0$ as $t \rightarrow-\infty$. By the translation invariant of (34) to (39) in $t$, we obtain the instability of the solutions of (34) to (39), i.e., the solutions will exit a small neighborhood of zero no matter how small the initial conditions are. After transforming (34) to (39) back to (1) to (5), we can see that the solutions will eventually leave the neighborhood of the steady state although the initial conditions are very close to the steady state. Thus we have the following instability results. 
Theorem 7. Let $\Omega^{*}, H_{p}^{k}\left(\Omega^{*}\right), U_{0}^{*}$, and $p_{0}^{*}$ be the same ones defined in Theorem 6 . If $\mathcal{A}$ in (51) has eigenvalues with positive real part, then there is a fixed number $\delta_{1}>0$ and an integer $k>1$ such that for any sufficiently small $\varepsilon>0$ there exists a $T_{\varepsilon}>0$ and an initial condition $\left(\zeta_{\varepsilon 0}^{*}, \underline{q}_{\varepsilon 0}^{*}, p_{\varepsilon 0}^{*}\right)$ satisfying the compatibility conditions and

$$
\left\|\zeta_{\varepsilon 0}^{*}\right\|_{H_{p}^{k+1 / 2}(\Gamma)}+\left\|\underline{q}_{\varepsilon 0}^{*}-U_{0}^{*}\right\|_{H_{p}^{k}\left(\Omega^{*}\right)}+\left\|p_{\varepsilon 0}^{*}-p_{0}^{*}\right\|_{H_{p}^{k-1}\left(\Omega^{*}\right)} \leq \varepsilon .
$$

Moreover, there is a solution $\left(\zeta_{\varepsilon}^{*}, \underline{q}_{\varepsilon}^{*}, p_{\varepsilon}^{*}\right)$ of the equations (1) to (5) for $t \in(0, T)$, satisfying the initial condition at $t=0$ and

$$
\left\|\zeta_{\varepsilon}^{*}\left(\cdot, T_{\varepsilon}\right)\right\|_{H_{p}^{5 / 2}(\Gamma)}+\left\|\underline{q}_{\varepsilon}^{*}\left(\cdot, T_{\varepsilon}\right)-U_{0}^{*}\right\|_{H_{p}^{2}\left(\Omega^{*}\right)}+\left\|p_{\varepsilon}^{*}\left(\cdot, T_{\varepsilon}\right)-p_{0}^{*}\right\|_{H_{p}^{1}\left(\Omega^{*}\right)} \geq \delta_{1}
$$

Acknowledgment. The author is grateful to Professor J. T. Beale for providing the most recent results of the problem considered in this paper. He is also thankful to the reviewer for his comments to improve the presentation of the paper.

\section{REFERENCES}

[1] T. B. Benjamin, Wave formation in laminar flow down an inclined plane, J. Fluid Mech. 2, 554-574 (1957)

[2] C.-S. Yih, Stability of liquid flow down an inclined plane, Phys. Fluids 6, 321-334 (1963)

[3] S. P. Lin, Instability of a liquid film flowing down an inclined plane, Phys. Fluids 10, 308-313 (1967)

[4] G. J. De Bruin, Stability of a layer of liquid flowing down an inclined plane, J. Engrg. Math. 8, 259-270 (1974)

[5] J. M. Floryan, S. H. Davis, and R. E. Kelly, Instabilities of a fluid film flowing down a slightly inclined plane, Phys. Fluids 30, 983-989 (1987)

[6] S. Chandrasekhar, Hydrodynamics and hydromagnetic stability, Oxford University Press, London, 1961

[7] C. C. Lin, The theory of hydrodynamic stability, Cambridge University Press, London, 1955

[8] D. H. Sattinger, The mathematical problem of hydrodynamic stability, J. Math. Mech. 19, 797-817 (1970)

[9] V. I. Yudovich, The linearization method in hydrodynamical stability theory, Translations of Mathematical Monographs, vol. 74, Amer. Math. Soc., Providence, Rhode Island, 1989

[10] J. Serrin, On stability of viscous fluid motions, Arch. Rational Mech. Anal. 3, 1-13 (1959)

[11] D. J. Benney, Long waves on liquid films, J. Math. Phys. 45, 150-155 (1966)

[12] M. C. Shen, S. M. Sun, and R. E. Meyer, Surface waves on viscous magnetic fluid flow down an inclined plane, Phys. Fluids A 3, 439-445 (1991)

[13] S. M. Shih and M. C. Shen, Uniform asymptotic approximation for viscous fluid flow down an inclined plane, SIAM J. Math. Anal. 6, 560-582 (1975)

[14] J. T. Beale, Large-time regularity of viscous surface waves, Arch. Rat. Mech. Anal. 84, 307-352 (1984)

[15] J. T. Beale and T. Nishida, Large-time behavior of viscous surface waves, Recent topics in Nonlinear PDE, II, North-Holland Math. Stud. 128, North-Holland, Amsterdam-New York, 1985, pp. $1-14$

[16] D. D. Joseph, Stability of fluid motions I, Springer-Verlag, New York, 1976

[17] M. Renardy and D. D. Joseph, Hopf bifurcation in two-component flow, SIAM J. Math. Anal. 17, 894-910 (1986)

[18] Y. Teramoto, On the Navier-Stokes flow down an inclined plane, J. Math. Kyoto Univ. 32, 593-619 (1992)

[19] T. Nishida, Y. Teramoto, and H. A. Win, Navier-Stokes flow down an inclined plane: Downward periodic motion, preprint 